\title{
Genetical Studies of Yield and its Components in Durum Wheat Under Heat Stress
}

Mostafa, M.O. $^{1}$; A.A. Ismail ${ }^{2}$; A. Abo-Elwafa ${ }^{2}$ and M.B. Tawfelis ${ }^{1}$

${ }^{1}$ Wheat Crops Res. Dept., Field Crops. Res. Inst., Agric. Res. Center, Giza-Egypt.

${ }^{2}$ Dept. of Agronomy, Assiut Univ., Assiut- Egypt.

\begin{abstract}
:
The present study was carried out at Assiut Agricultural Research Station during 2007/2008, 2008/2009, 2009/2010 seasons. Diallel cross without reciprocals among eight parents of durum wheat namely, Sohag $3\left(\mathrm{P}_{1}\right)$, line \#1 $\left(\mathrm{P}_{2}\right)$, line\# $2\left(\mathrm{P}_{3}\right)$, Bin- Sweef $1\left(\mathrm{P}_{4}\right)$, Bin- Sweef $5\left(\mathrm{P}_{5}\right)$, Karifla $\left(\mathrm{P}_{6}\right)$, Altar $84\left(\mathrm{P}_{7}\right)$ and Admor $\left(\mathrm{P}_{8}\right)$ were used to estimate heterosis, and general and specific combining ability under recommended and late planting dates. Mean squares showed that highly significant differences among genotypes, parents and crosses for all studied traits in the $\mathrm{F}_{1}$ 's and $\mathrm{F}_{2}$ generations, except number of spike/plant under late planting date. Moreover, both general (GCA) and specific (SCA) combining abilities were highly significant for most studied traits in the $F_{1}$ 's and $F_{2}$ generations under both planting dates except grain yield/plant, biological and number of kernel/spike in the $\mathrm{F}_{1}$ 's under late planting date in the SCA . The estimates of heterosis for grain yield/plant indicated that seven crosses out of $28 \mathrm{~F}_{1}$ 's hybrids significantly surpassed their better parent with percentage ranged from $8.798\left(\mathrm{P}_{4} \times \mathrm{P}_{7}\right)$ to $41.22 \%\left(\mathrm{P}_{1} \times \mathrm{P}_{6}\right)$. These relatively high heterotic percentage along with the variability existed among all diallel set increase the chance of good recombination's that can be isolated in the following generations particularly, when selfing in the following generations gives an essentially homozygous state and enhances the role of selected plants in reducing the effects of dominance. However, the additive gene effect was of great importance in the performance of the most studied trait i.e. No. of spikes/plant, grain yield/plant (g), biological yield/plant (g), number kernel/spike. Moreover, the parents $\mathrm{P}_{7}$ and $\mathrm{P}_{4}$ were good combiners for four traits i.e. Number of spikes/plant, grain yield/plant (g), biological yield/plant (g), number kernel/spike while, $\mathrm{P}_{2}$ was the best for only three traits i.e. days to heading, maturity and number kernel/spike date in the $\mathrm{F}_{1}$ 's and $\mathrm{F}_{2}$ generations. Meanwhile, significant SCA effects for grain yield were found in four out of the 28 $\mathrm{F}_{1}$ 's crosses $\mathrm{P}_{1} \times \mathrm{P}_{4}, \mathrm{P}_{1} \times \mathrm{P}_{6}, \mathrm{P}_{4} \times \mathrm{P}_{7}$ and $\mathrm{P}_{6} \times \mathrm{P}_{8}$ giving positive values of SCA effects under normal planting date and one cross $\mathrm{P}_{2} \times \mathrm{P}_{4}$ under late planting date. On the other view, $\mathrm{F}_{2}$ populations, one crosses $\mathrm{P}_{3} \times \mathrm{P}_{8}$ gave positive values of SCA effects .while in the $\mathrm{F}_{1}$ 'sand $\mathrm{F}_{2}$ generations, only one cross; $\mathrm{P}_{3} \times \mathrm{P}_{8}$ gave positive values of SCA effects for 100- kernel weight under both planting date.
\end{abstract}

Keywords: Diallel, crosses, durum wheat, heterosis, combining ability (GCA \& SCA).

Received on: 24/12 / 2014

Referees: Prof. Adel M. Mahmoud
Accepted for publication on: 13/1/2015

Prof. Mohamed A. Ali 


\section{Introduction:}

Wheat is one of the most important food crops in the world. The cultivated area in Egypt in 2012/2013 reached 3.5 million feddans with an average yield of $18.00 \mathrm{ardab} /$ feddan. The production was about 9.475 million Metric Tons, (FAO, 2013).

However, it is concentrated in relatively small geographical areas where it often plays a major role in the food security of urban population and in the livelihood and nutrition of urban communities. The productivity of durum wheat is often limited by an array of abiotic stresses that affect a successful growth and a complete grain filling. Heat stress, due to increase temperature, is an agricultural problem in many areas in the world (Wahid et al., 2007). A successful breeding program needs continuous information on the genetic variation and systems governing grain yield and its components. Tawfelis (1997), Hamada and Tawfelis (2001), Abd El-Majeed, et al. (2004) and El-Sayed (2004) showed that both additive and non-additive gene effects controlled the genetic system number of spikes/plant, number of kernels/spike, kernel weight and grain yield/plant. Uma and Sharma (1997) stated that SCA components of variance were higher than GCA in the inheritance of most of studied traits. Nayeem (1994) reported that both additive and nonadditive variation had important role in controlling the inheritance of days to flower and maturity date. Karrar (1980) found significant heterosis in durum wheat for grain yield, No. of spikes/plant, No of kernels/spike, kernel weight, plant height and days to heading comparing to better parent. Meanwhile, Hamada and Tawfe- lis (2001) reported that heterosis percentage based on better parent varied from -24.09 to $12.36,-15.00$ to 33.09 , -17.15 to $33.52,-35.01$ to 53.63 and from -24.15 to $72.16 \%$ for plant height, number of spikes/plant, number of kernels/spike, kernels/weight and grain yield/plant, respectively. Abd-Abdel-Kader (2006) found mean square showed that the highly significant differences among genotypes, parents and crosses for all studied traits in the $F_{1}$ and $F_{2}$ generations. Moreover, both general and specific combining ability were significant for all studied traits in the $\mathrm{F}_{1}$ and $\mathrm{F}_{2}$ generations. Akicnci (2009) found heterosis percentages for high- parent and mid- parent were $-2.16 \%$ and $-0.74 \%$ for heading date; $-1.64 \%$ and $3.78 \%$ for 1000 kernel weight; $-2.24 \%$ and $5.24 \%$ for plant yield, respectively. Moreover, both general and specific combining ability were significant for all studied traits. Irshad et al. (2012) found mean squares that highly significant differences among genotype, for days to heading, spike per plant, grain yield per plant. The present investigation was undertaken to were used estimate heterosis, general and specific combining ability in some durum wheat genotype.

Materials and Methods:

This study was conducted at Assiut Agricultural Research Station, ARC, Egypt during the three growing seasons of 2007/08, 2008/09 and 2009/010. The Genetical materials chosen to be used in this study as parents included eight durum wheat cultivars, which represents a wide range of diversity for several traits. The local name, pedigree and origin of these eight varieties are presented in Table (1). 
Table (1): Local name, pedigree and origin of the eight parents.

\begin{tabular}{|c|c|c|c|}
\hline & Name & Pedigree & Origin \\
\hline P1 & Sohag 3 & $\begin{array}{l}\text { MEXI "S" /MGHA/51792//DURUM } 6 \\
\text { CD21831-2sh-osh }\end{array}$ & Egypt \\
\hline $\mathbf{P 2}$ & Line $\neq 1$ & $\begin{array}{l}\text { SOOTY-9/RASCON-37. } \\
\text { CD91B1938-6M-O3OY-O3OM-4Y-OM-OB-1Y-OB-OSH }\end{array}$ & Egypt \\
\hline $\mathbf{P 3}$ & Line $\neq 2$ & $\begin{array}{l}\text { BOOMER-21/BUSCA-3 } \\
\text { CDSS95YOO1185-8Y-OM-OY-OB-IY-OB-OSD }\end{array}$ & Egypt \\
\hline $\mathbf{P 4}$ & Bani-Sweef 1 & $\begin{array}{l}\text { JO"S" / AA"S" // FG =BITTERN "S" } \\
\text { CD9799 }\end{array}$ & Egypt \\
\hline P5 & Bani-Sweef 5 & $\begin{array}{l}\text { Dipperz / bushen3 } \\
\text { CDSS92B128-1M-0Y-0M-0Y-3B-0Y-0SD }\end{array}$ & Egypt \\
\hline P6 & Karifla & Shandweel durum wheat breeding program & \\
\hline P7 & Altar84 & $\begin{array}{l}\text { Ruff "S" / FG "S" //MEXI } \\
\text { 75/3/SHWA "S" = GA"S" }\end{array}$ & Mexico \\
\hline P8 & Edmor & Edm. & USA \\
\hline
\end{tabular}

Table (2): Physical and chemical characteristics of representative composite soil sample from the field experimental site.

\begin{tabular}{|c|c|}
\hline Soil Properties & Values \\
\hline \multicolumn{2}{|l|}{ Particle size distribution } \\
\hline Sand $(\%)$ & 96.72 \\
\hline Silt $(\%)$ & 2.12 \\
\hline Clay $(\%)$ & 1.16 \\
\hline Soil texture & Sandy \\
\hline Field capacity (\%) & 9.92 \\
\hline Water saturation $(\%)$ & 20.58 \\
\hline Total $\mathrm{CaCO}_{3} \%$ & 35.18 \\
\hline EC mmhos/cm $(1: 1)$ & 0.35 \\
\hline $\mathrm{pH}$ ( $1: 1$ water suspension) & 8.65 \\
\hline Organic matter $\%$ & 0.24 \\
\hline \multicolumn{2}{|l|}{ Soluble cations $(\mathrm{meq} / \mathrm{L})$ : } \\
\hline $\mathrm{Ca}^{++}$ & 1.73 \\
\hline $\mathrm{Mg}^{++}$ & 1.00 \\
\hline $\mathrm{Na}^{+}$ & 0.56 \\
\hline $\mathrm{K}^{+}$ & 0.17 \\
\hline \multicolumn{2}{|l|}{ Soluble anions $(\mathrm{meq} / \mathrm{L})$} \\
\hline$+\mathrm{HCO}_{3} \mathrm{CO}_{3}$ & 1.70 \\
\hline $\mathrm{Cl}$ & 1.34 \\
\hline Total nitrogen $(\%)$ & 0.003 \\
\hline Available Phosphorus (ppm) & 8.30 \\
\hline
\end{tabular}


Table (3): Average of temperature through the growing seasons 2009/2010

\begin{tabular}{|c|c|c|c|c|}
\hline \multicolumn{2}{|c|}{ Season } & \multicolumn{3}{|c|}{$2009 / 2010$} \\
\hline Month & Day & Maximum & Minimum & Average \\
\hline & $1-10$ & 23.70 & 5.40 & 14.55 \\
\hline & $11-20$ & 24.90 & 7.70 & 16.30 \\
\hline January & $21-31$ & 23.36 & 4.70 & 14.03 \\
\hline \multicolumn{2}{|c|}{ Average } & 23.99 & 5.93 & 14.96 \\
\hline & $1-10$ & 19.40 & 5.00 & 12.20 \\
\hline & $11-20$ & 27.25 & 10.20 & 18.73 \\
\hline February & $21-28$ & 24.00 & 9.37 & 16.69 \\
\hline \multicolumn{2}{|c|}{ Average } & 23.55 & 8.19 & 15.87 \\
\hline \multirow{3}{*}{ March } & $1-10$ & 29.00 & 9.80 & 19.40 \\
\hline & $11-20$ & 27.20 & 14.60 & 20.90 \\
\hline & $21-31$ & 24.63 & 8.36 & 16.50 \\
\hline \multicolumn{2}{|c|}{ Average } & 26.94 & 10.92 & 18.93 \\
\hline \multirow{3}{*}{ April } & $1-10$ & 31.00 & 11.60 & 21.30 \\
\hline & $11-20$ & 33.80 & 13.00 & 23.40 \\
\hline & $21-30$ & 28.10 & 13.50 & 20.80 \\
\hline \multicolumn{2}{|c|}{$\frac{1}{\text { Average }}$} & 30.97 & 12.70 & 21.83 \\
\hline & $1-10$ & 35.08 & 18.46 & 26.77 \\
\hline & $11-20$ & 35.40 & 16.12 & 25.76 \\
\hline May & 21.31 & 38.10 & 19.06 & 28.58 \\
\hline \multicolumn{2}{|c|}{ Average } & 36.19 & 17.88 & 27.04 \\
\hline
\end{tabular}

In 2007/2008 season, a half diallel cross were made among the eight parents to produce 28 crosses. In 2008/2009 season, ten seeds from each cross were grown to produce seeds of $\mathrm{F}_{2}$ population. The eight parents were also grown for crossing to obtain more $\mathrm{F}_{1}$ 's hybrid seeds. In $2009 / 2010$ season the eight parents, 28 hybrids and $28 \quad \mathrm{~F}_{2}$ populations were grown in a randomized completely block design (RCBD) with three replicates at two planting dates $25^{\text {th }}$ of Nov (normal planting date) and $25^{\text {th }}$ of Dec (late planting date). Each of the parental materials were represented by six rows per replicate, while for $F_{1}$ 's crosses represented by one row of plants per replicate.

Each population was represented by six rows per bloke. The seeds were grown in 3 meter long rows spaced $30 \mathrm{~cm}$ apart and plant spaced $10 \mathrm{~cm}$, within each row. The cultural practices were applied as recommended and weeds were controlled by hand.

\section{Data collected}

The data were recorded on ten guarded plants of each parent and F1 hybrid and 50 plants of each F2 population from each replicate to measure the following characters:1- days to heading (days) 2- days to maturity (days) 3- number of spikes/plant,4biological yield/plant (g), 5- Grain yield/plant (g), 6- 100-kernel weight (g) and 7-number of kernels/spike

\section{Heterosis}

Heterosis was calculated as the percentage of deviation of $F_{1}$ 's mean from the mean of mid-parents and better parent according to following formula computed by Bhatt (1971): Heterosis relative to mid-parents 


$$
\mathrm{H}=\left[\left(\overline{\mathrm{F}}_{1}{ }^{\prime} \mathrm{s}-\overline{\mathrm{M} . \mathrm{P} .}\right) / \overline{\mathrm{M} . \mathrm{P} .}\right] \times 100
$$

Heterosis relative to better-parent $\mathrm{H}=\left[\left(\overline{\mathrm{F}}_{1}{ }_{1} \mathrm{~s}-\overline{\mathrm{B} . \mathrm{P} .}\right) / \overline{\mathrm{B} . \mathrm{P} .}\right] \times 100$

Where;

$\overline{\mathrm{F}}_{1}{ }^{\prime} \mathrm{s}=$ Mean of a $\mathrm{F}_{1}$ 's cross.

$\overline{\text { B.P. }}=$ Mean of the better parent.

$\overline{\text { M.P. }}=$ mean of mid-parents

To detect the significance of heterosis, the least significance differences (L.S.D) value from zero can be calculated as follows:

L.S.D of mid- parent heterosis = S.E. $\mathrm{x} t \alpha / \mathrm{MP}$

L.S.D of better parent heterosis =

S.E. $x$ t $\alpha /$ B.P

Where: S.E. for better parent = $[3 \mathrm{MSE} / 2 \mathrm{r}]^{1 / 2}$

S.E. for better parent $=[2 \mathrm{MSE} / \mathrm{r}]^{1 / 2}$

$\mathrm{T} \alpha=$ tabulated value at the degree of freedom for the error.

$\mathrm{MSE}=$ mean squares for error.

$\mathrm{r}=$ number of replications

\section{Combining ability analysis}

Estimates of general (G.C.A.) and specific combining ability (S.C.A.) variances and their effects were calculated using ordinary method for analysis of variance in randomized complete block design. If the differences between genotypes were significant, further analysis for general and specific combining ability was made according to Griffing (1956), method 2 model 1.

\section{Results and Discussion:}

The analysis of variance for all the studied traits of the eight parents and their $28 \mathrm{~F}_{1}$ 's and $28 \mathrm{~F}_{2}$ populations grown at of the two planting dates are presented in (Table 4). The analysis of variance revealed that mean squares due to genotypes, parents and crosses were highly significant for all the studied traits for $F_{1}$ 's
\& $\mathrm{F}_{2}$ under both planting dates i.e. days to heading and maturity, No. of spike/plant biological yield/plant, grain yield, No. of kernel/spike and 100-kernels weight, except No. of spike/plant under late planting date. This indicts the wide genetic diversity among the parental materials used in the present study. Also, mean squares due to $\mathrm{P}$. vs. C were highly significant for days to heading and maturity, grain yield/plant and number of kernel/spike. Mean squares due to No. of spike/plant were highly significant, except for $\mathrm{F}_{1}$ 's under normal planting date. The significant trend found to be with grain yield, except for $F_{1}$ 's under late planting date. Similar results were obtained by AlKoddoussi and Hassan (1991), Tawfelis (1997), Tammam and Abd ElGawad (1999), Ashoush et al. (2001), Moustafa (2002) and Abdel -kader (2006).

Mean squares due to GCA and SCA were highly significant for all studied traits under $F_{1}$ 's \& $F_{2}$ generations at both planting dates. Except grain yield/plant, biological yield/plant and no. of kernel/spike for $\mathrm{F}_{1}$ 's under late planting date (table 4). GCA/SCA ratios in $F_{1}$ 's \& $F_{2}$ were high values for biological yield/plant, grain yield/plant and number of kernel/spike under both planting dates (table 4). This indicates that additive genetic effect played the major rate in the inheritance for these traits- On the other hand, GCA/SCA ratios were very small for some studied traits in both generations $\left(\mathrm{F}_{1}\right.$ 's \& $\left.\mathrm{F}_{2}\right)$, reflecting that the non- additive effects contributes more important role than additive effects. The present finding were partially in harmony with those obtained by Mosaad et al. (1990), AlKoddoussi and Hassan (1991), Taw- 
felis (1997), Uma and Sharma (1997), Ashoush et al. (2001), Ismail et al. (2001)), Tawfelis et al. (2006), ElKaramity et al. (2007) and Akicnci (2009).

\section{Heterosis}

Heterosis values over mid and better parent for the studied traits are given in Table (5).

Day to healing estimates of heterosis for days to heading over the mid-parents (MP) and better parent (BP) under the two environments are presented in (Table 5).

Under normal planting date: there are thirteen and two crosses significantly earliar than its MP and $\mathrm{BP}$ respectively. The earlist crosses were $\left(\mathrm{P}_{6} \times \mathrm{P}_{8}\right)$ and $\left(\mathrm{P}_{3} \times \mathrm{P}_{6}\right)$ from MP and $\mathrm{BP}$ respectively its gave -9.615 and $-2.273 \%$ of heterosis, respectively

Under late planting date: there are thirteen and three crosses significantly ear liar than its MP and BP respectively. the ear list crosses were $\left(\mathrm{P}_{4} \times \mathrm{P}_{8}\right)$ and $\left(\mathrm{P}_{6} \times \mathrm{P}_{7}\right)$ from MP and $\mathrm{BP}$ respectively its gave -13.648 and $-5.333 \%$ of heterosis, respectively. Early flowering crosses can be used in breeding program for getting early flowering lines by different selection methods. These results agree with those reported by Zaied (1995), El-Sayed (1997), Twfelis (1997), Tammam and Abdel- Gawad (1999), Ashoush et al. (2001), AbdelHameed (2002), Mohamed (2007) and Akicnci (2009)

Day to maturity estimates of heterosis for days to maturity over the mid-parents and better parent under the two environments are presented in (Table 5).

Under normal planting date: there are nine and five crosses significantly earliar than its MP and BP respectively, the earliest crosses were $\left(\mathrm{P}_{4} \times \mathrm{P}_{6}\right)$ from MP and $\mathrm{BP}$ respectively, its gave -6.227 and $-5.185 \%$ of heterosis respectively. 
Table (4): Mean squares of combining ability analysis in of wheat genotypes under recommended $(\mathrm{N})$ and late $(\mathrm{L})$ planting dates.

\begin{tabular}{|c|c|c|c|c|c|c|c|c|c|}
\hline \multirow{3}{*}{$\begin{array}{l}\text { Source of } \\
\text { variance }\end{array}$} & \multirow{3}{*}{ Df } & \multicolumn{4}{|c|}{ Days to heading } & \multicolumn{4}{|c|}{ Days to maturity } \\
\hline & & \multicolumn{2}{|c|}{$F_{1}$} & \multicolumn{2}{|c|}{$\mathbf{F}_{2}$} & \multicolumn{2}{|c|}{$F_{1}$} & \multicolumn{2}{|c|}{$\mathbf{F}_{2}$} \\
\hline & & $\mathrm{N}$ & $\mathrm{L}$ & $\mathrm{N}$ & $\mathrm{L}$ & $\mathrm{N}$ & $\mathrm{L}$ & $\mathrm{N}$ & $\mathrm{L}$ \\
\hline Reps (R) & 2 & 2.62 & 6.95 & 8.62 & 6.86 & 2.25 & 1.18 & 2.26 & 1.95 \\
\hline Genotypes $(\mathrm{G})$ & 35 & $91.71 * *$ & $119.50 * *$ & $121.40 * *$ & $119.70 * *$ & $43.15 * *$ & $66.78 * *$ & $73.96^{* *}$ & $96.35^{* *}$ \\
\hline Parents (P) & 7 & $349.70 * *$ & $422.50 * *$ & $349.70 * *$ & $422.50 * *$ & $70.93 * *$ & $138.80^{* *}$ & $70.93 * *$ & $138.80 * *$ \\
\hline Crosses (C) & 27 & $27.30 * *$ & $40.50^{* *}$ & $55.52 * *$ & $40.78^{* *}$ & $36.59 * *$ & $49.57 * *$ & $69.24 * *$ & $87.65 * *$ \\
\hline $\mathrm{PxC}$ & 1 & $24.85^{* *}$ & $131.50^{* *}$ & $302.06^{* *}$ & $130.94^{* *}$ & $25.81 * *$ & $27.31^{* *}$ & $222.61 * *$ & $34.10^{* *}$ \\
\hline Error & 70 & 1.239 & 0.87 & 0.9 & 1.75 & 1.12 & 1.3 & 1.107 & 1.28 \\
\hline GCA & 7 & $309.22 * *$ & $415.30^{* *}$ & $429.50 * *$ & $408.60 * *$ & $147.20 * *$ & $183.40 * *$ & $234.30 * *$ & $312.40 * *$ \\
\hline SCA & 28 & $37.33 * *$ & $45.51 * *$ & $44.37 * *$ & $47.51 * *$ & $17.14 * *$ & $46.60 * *$ & $24.89^{* *}$ & $42.33 * *$ \\
\hline Error & 70 & 1.239 & 0.87 & 0.9 & 1.75 & 1.12 & 1.3 & 1.11 & 1.28 \\
\hline GCA/SCA & & 0.85 & 0.93 & 0.99 & 0.89 & 0.91 & 0.40 & 0.98 & 0.76 \\
\hline
\end{tabular}

\begin{tabular}{|l|c|c|c|c|c|}
\hline \multirow{2}{*}{ Source of variance } & \multirow{2}{*}{$\mathbf{d f}$} & \multicolumn{3}{|c|}{ No. of spike / plant } & \multicolumn{2}{c|}{ No. of spike / plant } \\
\cline { 3 - 7 } & & \multicolumn{2}{|c|}{$\mathbf{F}_{\mathbf{1}}$} & \multicolumn{2}{c|}{$\mathbf{F}_{\mathbf{2}}$} \\
\cline { 3 - 7 } & $\mathbf{2}$ & 2.84 & 0.039 & 2.25 & 1.02 \\
\hline Reps (R) & $\mathbf{3 5}$ & $5.94^{* *}$ & $1.31^{* *}$ & $5.88^{* *}$ & $1.15^{*}$ \\
\hline Genotypes(G) & $\mathbf{7}$ & $9.77^{* *}$ & $3.21^{* *}$ & $9.77^{* *}$ & $3.21^{*}$ \\
\hline Parents $(\mathrm{P})$ & $\mathbf{2 7}$ & $5.16^{* *}$ & 0.32 & $4.25^{* *}$ & 0.33 \\
\hline Crosses $(\mathrm{C})$ & $\mathbf{1}$ & 0.19 & $14.39^{* *}$ & $22.26^{* *}$ & $9.14^{* *}$ \\
\hline PxC & $\mathbf{7 0}$ & 0.69 & 0.44 & 1.21 & 2.93 \\
\hline Error & $\mathbf{7}$ & $21.41^{* *}$ & $2.05^{* *}$ & $19.69^{* *}$ & $1.58^{* *}$ \\
\hline GCA & $\mathbf{2 8}$ & $2.07^{* *}$ & $1.11^{* *}$ & $2.43^{* *}$ & $1.04 * *$ \\
\hline SCA & $\mathbf{7 0}$ & 0.69 & 0.44 & 1.21 & 2.93 \\
\hline Error & & 1.5 & 0.24 & 1.51 & 0.17 \\
\hline GCA/SCA & & & & & \\
\hline
\end{tabular}


Count. Table (4): Mean squares of combining ability analysis of wheat genotypes under recommended $(\mathrm{N})$ and late $(\mathrm{L})$ planting dates.

\begin{tabular}{|c|c|c|c|c|c|c|c|c|c|}
\hline \multirow{3}{*}{$\begin{array}{l}\text { Source of } \\
\text { variance }\end{array}$} & \multirow{3}{*}{ Df } & \multicolumn{4}{|c|}{ Grain yield / plant (g) } & \multicolumn{4}{|c|}{ Biological yield / plant(g) } \\
\hline & & \multicolumn{2}{|c|}{$\mathbf{F}_{1}$} & \multicolumn{2}{|c|}{$\mathbf{F}_{2}$} & \multicolumn{2}{|c|}{$\mathbf{F}_{1}$} & \multicolumn{2}{|c|}{$\mathbf{F}_{2}$} \\
\hline & & $\mathbf{N}$ & $\mathbf{L}$ & $\mathbf{N}$ & $\mathbf{L}$ & $\mathbf{N}$ & $\mathbf{L}$ & $\mathbf{N}$ & $\mathbf{L}$ \\
\hline Reps (R) & 2 & 9.71 & 3.11 & 2.11 & 0.14 & 41.76 & 11.98 & 8.16 & 14.12 \\
\hline Genotypes (G) & 35 & $39.37 * *$ & $22.02 * *$ & $30.66^{* *}$ & $16.34 * *$ & $355.36^{* *}$ & $180.30^{* *}$ & $299.40 * *$ & $158.00 * *$ \\
\hline Parents (P) & 7 & $62.63 * *$ & $34.14 * *$ & $62.63 * *$ & $34.14 * *$ & $525.40 * *$ & $238.10^{* *}$ & $525.40^{* *}$ & $238.10 * *$ \\
\hline Crosses (C) & 27 & $33.39 * *$ & $19.69^{* *}$ & $8.10^{* *}$ & $10.36^{* *}$ & $322.40 * *$ & $170.10^{* *}$ & $51.59^{* *}$ & $117.70 * *$ \\
\hline $\mathrm{PxC}$ & 1 & $38.01 * *$ & 0.09 & $416.00 * *$ & $53.20 * *$ & 55.00 & 51.10 & $5408.00 * *$ & $685.40 * *$ \\
\hline Error & 70 & 4.73 & 3.62 & 2.86 & 3.37 & 37.84 & 41.46 & 18.92 & 24.75 \\
\hline GCA & 7 & $139.30 * *$ & $94.32 * *$ & $52.14 * *$ & $54.70^{* *}$ & $1178.20 * *$ & $729.00^{* *}$ & $360.70 * *$ & $484.70 * *$ \\
\hline SCA & 28 & $14.40 * *$ & 3.95 & $25.29 * *$ & $6.75^{* *}$ & $149.70^{*}$ & 43.15 & $284.06^{* *}$ & $76.29 * *$ \\
\hline Error & 70 & 4.73 & 3.62 & 2.86 & 3.37 & 37.84 & 41.46 & 18.92 & 24.75 \\
\hline GCA/SCA & & 1.39 & 27.99 & 0.22 & 1.52 & 1.02 & 40.68 & 0.016 & 0.89 \\
\hline
\end{tabular}

\begin{tabular}{|c|c|c|c|c|c|c|c|c|c|}
\hline \multirow{3}{*}{$\begin{array}{l}\text { Source of va- } \\
\text { riance }\end{array}$} & \multirow{3}{*}{ df } & \multicolumn{4}{|c|}{ Number kernel/spike } & \multicolumn{4}{|c|}{100 - kernel weight } \\
\hline & & \multicolumn{2}{|c|}{$\mathbf{F}_{1}$} & \multicolumn{2}{|c|}{$\mathbf{F}_{2}$} & \multicolumn{2}{|c|}{$\mathbf{F}_{1}$} & \multicolumn{2}{|c|}{$\mathbf{F}_{2}$} \\
\hline & & $\mathrm{N}$ & $\mathrm{L}$ & $\mathrm{N}$ & $\mathrm{L}$ & $\mathrm{N}$ & $\mathrm{L}$ & $\mathrm{N}$ & $\mathrm{L}$ \\
\hline Reps (R) & 2 & 69.5 & 33.81 & 59.76 & 4.92 & 0.126 & 0.148 & 0.527 & 0.092 \\
\hline Genotypes $(\mathrm{G})$ & 35 & $169.80^{* *}$ & $130.00 * *$ & $149.40^{* *}$ & $125.50 * *$ & $0.607 * *$ & $0.730 * *$ & $0.764 * *$ & $0.513 * *$ \\
\hline Parents $(\mathrm{P})$ & 7 & $339.80 * *$ & $277.20 * *$ & $339.80 * *$ & $277.20 * *$ & $1.796^{* *}$ & $0.919 * *$ & $1.796 * *$ & $0.919 * *$ \\
\hline Crosses $(\mathrm{C})$ & 27 & $132.00 * *$ & $92.95 * *$ & $89.21 * *$ & $81.25^{* *}$ & $0.286^{* *}$ & $0.482 * *$ & $0.515^{*}$ & $0.342 * *$ \\
\hline PxC & 1 & 0.4 & $99.95 * *$ & $441.73 * *$ & $258.35 * *$ & $0.957 * *$ & $6.106^{* *}$ & $0.253^{*}$ & $0.747 * *$ \\
\hline Error & 70 & 16.25 & 18.38 & 12.28 & 13.95 & 0.145 & 0.122 & 0.203 & 0.102 \\
\hline GCA & 7 & $686.80 * *$ & $465.70 * *$ & $479.00 * *$ & $480.40 * *$ & $1.579 * *$ & $1.457^{* *}$ & $2.328 * *$ & $2.307 * *$ \\
\hline SCA & 28 & $40.59 * *$ & 46.04 & $67.05 * *$ & $36.74 * *$ & $0.364 * *$ & $0.548 * *$ & $0.373 *$ & $0.254 * *$ \\
\hline Error & 70 & 16.25 & 18.38 & 12.28 & 13.95 & 0.145 & 0.122 & 0.203 & 0.102 \\
\hline GCA/SCA & & 2.76 & 1.62 & 0.85 & 2.05 & 0.65 & 0.314 & 1.25 & 0.95 \\
\hline
\end{tabular}


Table (5): Heterosis percentages from mid-parent (MP) and better parent (BP) heterosis for days to heading and days to maturity of $28 \mathrm{~F}_{1}$ 's crosses under recommended $(\mathrm{N})$ and late $(\mathrm{L})$ planting dates.

\begin{tabular}{|c|c|c|c|c|c|c|c|c|}
\hline \multirow{3}{*}{$F_{1}$ crosses } & \multicolumn{4}{|c|}{ Days to heading } & \multicolumn{4}{|c|}{ Days to maturity } \\
\hline & \multicolumn{2}{|c|}{$\mathrm{N}$} & \multicolumn{2}{|c|}{$\mathrm{L}$} & \multicolumn{2}{|c|}{$\mathrm{N}$} & \multicolumn{2}{|c|}{$\mathrm{L}$} \\
\hline & MP & BP & MP & BP & MP & $\mathrm{BP}$ & MP & $\mathrm{BP}$ \\
\hline $\mathrm{P}_{1} \mathrm{XP}_{2}$ & $4.762 * *$ & $024 * *$ & -0.662 & 1.351 & $1.946^{*}$ & $.150^{* *}$ & $.670 * *$ & $4.630 * *$ \\
\hline $\mathrm{P}_{1} \mathrm{xP}_{3}$ & $2.890 * *$ & $4.706^{* *}$ & $1.333^{*}$ & $4.110 * *$ & 0.608 & $1.769 *$ & $1.991 *$ & $4.352 * *$ \\
\hline $\mathrm{P}_{1} \mathrm{XP}_{4}$ & $-3.371 * *$ & 1.176 & $-7.595^{* *}$ & $-5.195 * *$ & $1.887^{*}$ & $3.846^{* *}$ & 6.25 & $10.185^{* *}$ \\
\hline $\mathrm{P}_{1} \mathrm{xP}_{5}$ & 0.770 & 3.14 & & & -0.375 & & -0.441 & \\
\hline $\mathrm{P}_{1} \mathrm{xP}_{6}$ & .375 & 3.91 & $128^{* *}$ & $-3.896 * *$ & $2.239 * *$ & $5.385 * *$ & $2.655 * *$ & $7.407 * *$ \\
\hline $\mathrm{P}_{1} \times \mathrm{P}_{7}$ & $5.649 * *$ & $6.271 * *$ & $4.829^{* *}$ & $6.227 * *$ & $3.475 * *$ & $3.876^{* *}$ & $5.505 * *$ & $6.481^{* *}$ \\
\hline $\mathrm{P}_{1} \mathrm{xP}_{8}$ & $-6.931 * *$ & 10.58 & $-11.828^{* *}$ & & $1.845^{*}$ & & 1266 & $1 * *$ \\
\hline $\mathrm{P}_{2} \mathrm{xP}_{3}$ & $7.602 * *$ & 10.8 & & & -0.769 & & $2+2$ & $3.636^{* *}$ \\
\hline $\mathrm{P}_{2} \mathrm{XP}_{4}$ & $-2.648 * *$ & $3.217 * *$ & $-5.806^{* *}$ & -1.351 & -0.763 & $2.362 * *$ & 0.000 & $2.727 * *$ \\
\hline $\mathrm{P}_{2} \mathrm{xP}_{5}$ & $2.709 * *$ & 6.42 & & 1.7 & $-2.500 * *$ & 1. & $* *$ & 09 \\
\hline & $3.069 * *$ & & & & & & & 00 \\
\hline $\mathrm{P}_{2} \mathrm{xP}_{7}$ & 650 & & & & & & & -1.182 \\
\hline $\mathrm{P}_{2} \mathrm{xP}_{8}$ & $-8.000 * *$ & 10.8 & -10 & 10.8 & 0. & $6.299 * *$ & -0.418 & $2 * *$ \\
\hline & $972 * *$ & & & & & & & \\
\hline $\mathrm{P}_{3} \mathrm{xP}_{5}$ & 1.69 & & $* *$ & $4.110^{* *}$ & $-3.481 * *$ & $-2.030^{*}$ & $-5.172 * *$ & $-2.655^{* *}$ \\
\hline $\mathrm{P}_{3} \mathrm{xP}_{6}$ & $-3.911 * *$ & & $-3.947 * *$ & 000 & $-2.804 * *$ & -0.977 & $-3.030 * *$ & -0.885 \\
\hline $\mathrm{P}_{3} \mathrm{xP}_{7}$ & & & & & & & & -1.182 \\
\hline $\mathrm{P}_{3} \mathrm{xP}_{8}$ & -8.2 & & $1 * *$ & 13. & -2.1 & 0.7 & & $3.540 * *$ \\
\hline $\mathrm{P}_{4} \mathrm{xP}_{5}$ & 0.736 & & & & $-3.676^{* *}$ & $-2.963 * *$ & $-2.979 * *$ & -1.724 \\
\hline $\mathrm{P}_{4} \mathrm{xP}_{6}$ & $-3.261 * *$ & & 1.250 & $2.532 * *$ & $-6.227 * *$ & $-5.185^{* *}$ & $-3.419 * *$ & $-2.586^{* *}$ \\
\hline $\mathrm{P}_{4} \mathrm{xP}_{7}$ & $559 * *$ & & & & -4. & & $5 * *$ & $6^{* * *}$ \\
\hline $\mathrm{P}_{4} \mathrm{xP}_{8}$ & $-9.524 * *$ & $2.151 *$ & $-13.684 * *$ & 5 & 0.725 & $2.963 * *$ & $-2.041^{*}$ & $3.448 * *$ \\
\hline $\mathrm{P}_{5} \mathrm{xP}_{6}$ & & & & & $-2.764 * *$ & $-2.409 * *$ & $2.700 * *$ & $3.136^{* *}$ \\
\hline $\mathrm{P}_{5} \mathrm{xP}_{7}$ & $00 * *$ & & & & 00 & 3.1 & $5 * *$ & 45 \\
\hline $\mathrm{P}_{5} \mathrm{xP}_{8}$ & $-6.796 * *$ & 7.8 & $3^{* *}$ & $7 * *$ & -1.439 & 00 & $2^{* *}$ & 0.000 \\
\hline $\mathrm{P}_{6} \mathrm{xP}_{7}$ & $-3.955^{* *}$ & -1.163 & $-13.415^{* *}$ & $-5.333 * *$ & $-3.371 * *$ & 0.000 & $-2.895 * *$ & 0.636 \\
\hline $\mathrm{P}_{6} \mathrm{xP}_{8}$ & $615 * *$ & & $66^{* *}$ & & -1.290 & -0.217 & $4^{* *}$ & 0.847 \\
\hline $\mathrm{P}_{7} \mathrm{xP}_{8}$ & $-9.034 * *$ & $7.360 * *$ & $-9.783 * *$ & $10.667 * *$ & 0.741 & 5.426 & $-1.841^{*}$ & $6.636 * *$ \\
\hline LSD 5\% & & & & & 1.490 & 1.721 & 1.608 & 1.856 \\
\hline & & & & & 1.979 & & 2.135 & 2.465 \\
\hline Average & 9 & 4.803 & -3 & 3.684 & 2 & 8 & 1 & 35 \\
\hline
\end{tabular}

*and **: significant at 0.05 and 0.01 levels of probability, respectively

Under late planting date : there are sixteen and three crosses significantly earliar than its MP and BP respectively the earliest crosses were $\left(\mathrm{P}_{4} \times \mathrm{P}_{7}\right)$ from MP and $\mathrm{BP}$ respectively, its gave -6.195 and $-3.636 \%$ of heterosis, respectively. Early maturity crosses can be used in breeding program for getting early maturity lines by different selection methods. These results agree with those reported by Zaied (1995), Abdel -kader (2006), and Mohamed (2007).

Estimates of heterosis for number of $n$. spikes/plant over midparents and better parent under the two environments are presented in (Table 6).

Under normal planting date: there are twenty eight and twenty one 
crosses significantly no. of spike / plant at MP and $\mathrm{BP}$ respectively. The tillering crosses were $\left(\mathrm{P}_{1} \times \mathrm{P}_{6}\right)$ from MP and BP, respectively, its gave 23.544 and $17.984 \%$ of heterosis, respectively.

Under late planting date: there are ten and eight crosses significantly no. of spike / plant at MP and BP respectively. The $\mathrm{n}$. of spike / plant crosses were $\left(\mathrm{P}_{6} \times \mathrm{P}_{7}\right)$ from MP and $\mathrm{BP}$, respectively its gave 16.972 and $16.555 \%$ of heterosis, respectively.

Eight hybrids reflected that showed highly significant positive heterosis under late planting date. It can be used in breeding program from tolerant to heat under late planting date by different selection methods. These results agree with those reported by Hamada and Twfelis (2001), Sharma et al. (2002) and Abdel -kader (2006).

Estimates of heterosis for grain yield/plant (Table 7) indicated that 19 and 7 crosses under normal planting date and 11 and 3 crosses under late planting date were significantly positive over the MP and BP, respectively. This indicted that two crosses $\left(\mathrm{P}_{2} \times \mathrm{P}_{7}\right)$ and $\left(\mathrm{P}_{4} \times \mathrm{P}_{7}\right)$ gives positive significant heterosis over MP and BP under both planting date. Variable amount of heterosis were found from planting date to another which could be highly significant interaction and parent vs. crosses. Furthermore, the sensitivity of the parent to heat stress which was one of the major causes of heterosis fluctuation. Heterosis for grain yield/plant (Table 5) ranged from $3.829 \quad\left(\mathrm{P}_{3} \times \mathrm{P}_{7}\right)$ to $55.491 \%$ $\left(\mathrm{P}_{1} \times \mathrm{P}_{6}\right)$ at $\mathrm{MP}$ and from 8.798 $\left(\mathrm{P}_{4} \times \mathrm{P}_{7}\right)$ to $41.122 \%\left(\mathrm{P}_{1} \times \mathrm{P}_{6}\right)$ at $\mathrm{BP}$ under normal planting date. While, under late planting date it ranged from $5.704\left(\mathrm{P}_{5} \times \mathrm{P}_{6}\right)$ to $17.980 \%$
$\left(\mathrm{P}_{2} \times \mathrm{P}_{4}\right)$ and from $4.297 \quad\left(\mathrm{P}_{2} \times \mathrm{P}_{7}\right)$ to $11.532 \%\left(\mathrm{P}_{2} \times \mathrm{P}_{4}\right)$ at $\mathrm{MP}$ and $\mathrm{BP}$ respectively. These results in agreement with those reported by Al-Kodoussi and Hassan (1995), Hamada and Twfelis (2001), Abdel-kader (2006) and Akicnci (2009).

With respect to the biological yield/plant (Table 7) indicated that six and one crosses under normal planting date 5 and one crosses under late planting date significantly positive over MP respectively. It is clear results that many of crosses possessed negative heterosis value over MP and BP even under normal or late planting date. Heterosis for biological yield/plant (Table 5) ranged from $9.587\left(\mathrm{P}_{1} \times \mathrm{P}_{8}\right)$ to $30.965 \%\left(\mathrm{P}_{1} \times \mathrm{P}_{6}\right)$ at MP under normal planting. While, under late planting ranged from 9.932 $\left(\mathrm{P}_{3} \times \mathrm{P}_{6}\right)$ to $22.718 \%\left(\mathrm{P}_{7} \times \mathrm{P}_{8}\right)$ at $\mathrm{MP}$ respectively. These results are in agreement with those reported by Zaied (1995) and Abd-Elkader (2006).

With regard to the number of kernel/spike (Table 8) indication that two crosses and normal planting at $\mathrm{Mp}$ and 13 and 3 crosses under late planting date were significantly positive over the MP and BP, respectively. Heterosis for number kernel/spike (Table 6) ranged from $11.445\left(\mathrm{P}_{1} \times \mathrm{P}_{3}\right)$ to $17.488 \%\left(\mathrm{P}_{3} \times \mathrm{P}_{4}\right)$ at $\mathrm{MP}$ under normal planting. Otherwise, there is no crosses had positive value over better parent. While, under late planting it ranged from $6.861\left(\mathrm{P}_{3} \times \mathrm{P}_{8}\right)$ to $24.147 \% \quad\left(\mathrm{P}_{6} \times \mathrm{P}_{8}\right)$ and from 7.273 $\left(\mathrm{P}_{6} \times \mathrm{P}_{8}\right)$ to $10.803 \%\left(\mathrm{P}_{2} \times \mathrm{P}_{6}\right)$ at $\mathrm{MP}$ and $\mathrm{BP}$, respectively table 6 . These results are in line with those reported by Al-Kodoussi and Hassan (1995), Hamada and Twfelis (2001), Ashoush (2002) and Nagwa (2007). 
Table (6): Heterosis percentages from mid-parent (MP) and better parent (BP) heterosis for No. of spike/plant of $28 \mathrm{~F}_{1}$ 's crosses under recommended $(\mathrm{N})$ and late $(\mathrm{L})$ planting dates.

\begin{tabular}{|c|c|c|c|c|}
\hline \multirow{3}{*}{ F1 crosses } & \multirow{2}{*}{\multicolumn{2}{|c|}{$\begin{array}{c}\text { No. of spike / plant } \\
\mathbf{N}\end{array}$}} & \multirow{2}{*}{\multicolumn{2}{|c|}{$\frac{\text { No. of spike / plant }}{\text { L }}$}} \\
\hline & & & & \\
\hline & MP & BP & MP & BP \\
\hline $\mathrm{P}_{1} \mathrm{xP}_{2}$ & $14.038^{* *}$ & $12.424^{* *}$ & $-2.830 * *$ & $-3.507 * *$ \\
\hline $\mathrm{P}_{1} \mathrm{xP}_{3}$ & $11.909 * *$ & $6.873 * *$ & $-22.404 * *$ & $-27.038 * *$ \\
\hline $\mathrm{P}_{1} \mathrm{xP}_{4}$ & $13.044 * *$ & $6.123 * *$ & $6.040 * *$ & 0.000 \\
\hline $\mathrm{P}_{1} \mathrm{xP}_{5}$ & $11.106^{* *}$ & $4.051 * *$ & $-21.140 * *$ & $-21.427 * *$ \\
\hline $\mathrm{P}_{1} \mathrm{xP}_{6}$ & $23.544 * *$ & $17.984 * *$ & $11.118^{* *}$ & $10.714 * *$ \\
\hline $\mathrm{P}_{1} \mathrm{xP}_{7}$ & $11.776^{* *}$ & $3.475^{* *}$ & $-9.356^{* *}$ & $-10.006^{* *}$ \\
\hline $\mathrm{P}_{1} \mathrm{xP}_{8}$ & $10.589 * *$ & -0.474 & $-0.708^{*}$ & $-2.083 * *$ \\
\hline $\mathrm{P}_{2} \mathrm{XP}_{3}$ & $8.738 * *$ & $5.286 * *$ & $-5.651 * *$ & $-10.698 * *$ \\
\hline $\mathrm{P}_{2} \mathrm{XP}_{4}$ & $11.526^{* *}$ & $6.123^{* *}$ & $11.340^{* *}$ & $5.696^{* *}$ \\
\hline $\mathrm{P}_{2} \mathrm{XP}_{5}$ & $2.142 * *$ & $-3.046^{* *}$ & $3.203 * *$ & $2.113 * *$ \\
\hline $\mathrm{P}_{2} \mathrm{XP}_{6}$ & $3.820 * *$ & 0.524 & $-18.151 * *$ & $-19.015^{* *}$ \\
\hline $\mathrm{P}_{2} \mathrm{xP}_{7}$ & $15.570 * *$ & $8.421 * *$ & $9.290 * *$ & $7.754 * *$ \\
\hline $\mathrm{P}_{2} \mathrm{XP}_{8}$ & $6.115^{* *}$ & $-3.265^{* *}$ & $-3.493 * *$ & $-4.167 * *$ \\
\hline $\mathrm{P}_{3} \mathrm{xP}_{4}$ & $11.167^{* *}$ & $9.184 * *$ & $-4.098 * *$ & $-4.396^{* *}$ \\
\hline $\mathrm{P}_{3} \mathrm{XP}_{5}$ & $3.629 * *$ & $1.523 * *$ & $-3.352 * *$ & $-9.434 * *$ \\
\hline $\mathrm{P}_{3} \mathrm{xP}_{6}$ & $5.286 * *$ & $5.286 * *$ & $-14.769 * *$ & $-20.132 * *$ \\
\hline $\mathrm{P}_{3} \mathrm{xP}_{7}$ & $2.816^{* *}$ & -0.490 & $-2.364 * *$ & $-8.811 * *$ \\
\hline $\mathrm{P}_{3} \mathrm{xP}_{8}$ & $5.933 * *$ & -0.474 & $-21.446^{* *}$ & $-25.151 * *$ \\
\hline $\mathrm{P}_{4} \mathrm{XP}_{5}$ & $5.847 * *$ & $5.573 * *$ & $-7.071 * *$ & $-12.664 * *$ \\
\hline $\mathrm{P}_{4} \mathrm{xP}_{6}$ & $11.696^{* *}$ & $9.705^{* *}$ & $2.364 * *$ & $-3.797 * *$ \\
\hline $\mathrm{P}_{4} \mathrm{xP}_{7}$ & $7.041 * *$ & $5.451^{* *}$ & $-2.037 * *$ & $-8.240 * *$ \\
\hline $\mathrm{P}_{4} \mathrm{xP}_{8}$ & $6.569 * *$ & $1.856^{* *}$ & $-10.599 * *$ & $-14.562 * *$ \\
\hline $\mathrm{P}_{5} \mathrm{xP}_{6}$ & $10.873 * *$ & $8.619 * *$ & $6.475 * *$ & $6.475 * *$ \\
\hline $\mathrm{P}_{5} \mathrm{xP}_{7}$ & $11.278^{* *}$ & $9.906^{* *}$ & $9.022 * *$ & $8.634 * *$ \\
\hline $\mathrm{P}_{5} \mathrm{xP}_{8}$ & $19.412 * *$ & $14.413 * *$ & $-6.710 * *$ & $-8.333 * *$ \\
\hline $\mathrm{P}_{6} \mathrm{xP}_{7}$ & $17.133 * *$ & $13.367 * *$ & $16.972 * *$ & $16.555 * *$ \\
\hline $\mathrm{P}_{6} \mathrm{xP}_{8}$ & $7.418 * *$ & $0.921 *$ & $-10.251 * *$ & $-11.813^{* *}$ \\
\hline $\mathrm{P}_{7} \mathrm{xP}_{8}$ & $3.597 * *$ & 0.460 & $11.340^{* *}$ & $9.021 * *$ \\
\hline LSD $5 \%$ & 0.742 & 0.857 & 0.613 & 0.708 \\
\hline LSD 1\% & 0.985 & 1.138 & 0.814 & 0.940 \\
\hline $\begin{array}{l}\text { Average hetero- } \\
\text { sis }\end{array}$ & 9.772 & 5.350 & -2.831 & -5.654 \\
\hline
\end{tabular}

*and **: significant at 0.05 and 0.01 levels of probability, respectively 
Table (7): Heterosis percentages from mid-parent (MP) and better parent (BP) heterosis for grain yield/ plant and biological yield/plant of $28 F_{1}$ 's crosses, under recommended $(N)$ and late $(L)$ planting dates.

\begin{tabular}{|c|c|c|c|c|c|c|c|c|}
\hline \multirow{3}{*}{$\begin{array}{c}\text { F1 } \\
\text { crosses }\end{array}$} & \multicolumn{4}{|c|}{ Grain yield / plant (g) } & \multicolumn{4}{|c|}{ Biological yield / plant(g) } \\
\hline & \multicolumn{2}{|c|}{$\mathrm{N}$} & \multicolumn{2}{|c|}{ 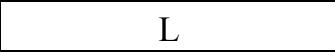 } & & \multicolumn{2}{|c|}{$\mathrm{I}$} \\
\hline & I & & & BP & & & & BP \\
\hline $\mathrm{P}_{1} \mathrm{xP}_{2}$ & $690 * *$ & & & $0.317^{* *}$ & $-11.517^{*}$ & & & $-15.392^{*}$ \\
\hline $\mathrm{P}_{1} \times \mathrm{P}_{3}$ & $*$ & 234 & $-11.632 * *$ & $5.449 * *$ & 4.318 & & -16.0 & -3 \\
\hline $\mathrm{P}_{1} \mathrm{xP}_{4}$ & & & & & $6.546 * *$ & & & $26^{* *}$ \\
\hline $\mathrm{P}_{1} \times \mathrm{P}_{5}$ & & & & $-17.613^{* *}$ & $-11.429 * *$ & & & $619 * *$ \\
\hline $\mathrm{P}_{1} \mathrm{xP}_{6}$ & $0 \%$ & $* *$ & 24 & $5.521 * *$ & $* *$ & & & \\
\hline $\mathrm{P}_{1} \mathrm{xP}_{7}$ & & & & & & & & 774 \\
\hline $\mathrm{P}_{1} \mathrm{xP}_{8}$ & & ** & \begin{tabular}{|l|} 
\\
\end{tabular} & .876 & & 49 & & 4.898 \\
\hline $\mathrm{P}_{2} \mathrm{XP}_{3}$ & & & & & & & & \\
\hline & & & & & & & & \\
\hline $\mathrm{P}_{2} \mathrm{XP}_{5}$ & & -18.2 & -12 & -18 & $-25.942 * *$ & & -13 & $-24.194 * *$ \\
\hline & & & & & & & & \\
\hline & & & & & & & & \\
\hline $\mathrm{P}_{2} \mathrm{XP}_{8}$ & & -10 . & - & $-15.509 * *$ & -5.215 & & $-12.103 * *$ & $-20.264 * *$ \\
\hline & & & & & $-14.477 * *$ & & & $-10.693^{*}$ \\
\hline & & & & & & & & \\
\hline $\mathrm{P}_{3} \mathrm{xP}_{6}$ & & & & & & & & \\
\hline & & & & & & & & $-14.044 * *$ \\
\hline $\mathrm{P}_{3} \mathrm{xP}_{8}$ & & & & & & & & \\
\hline & & & & & & & & \\
\hline $\mathrm{P}_{4} \mathrm{xP}_{6}$ & & & & & $-25.072 * *$ & & & $63 * *$ \\
\hline $\mathrm{P}_{4} \mathrm{xP}_{7}$ & & & $* *$ & $7.568 * *$ & $11.836^{* *}$ & 51 & & 35 \\
\hline $\mathrm{P}_{4} \mathrm{xP}_{8}$ & & & & $-23.303 * *$ & $-22.450 * *$ & $39.164 * *$ & & \\
\hline $\mathrm{P}_{5} \mathrm{xP}_{6}$ & & & & & & & & $-23.467 * *$ \\
\hline $\mathrm{P}_{5} \mathrm{xP}_{7}$ & ** & -3 & 6 & -9.7 & -16 . & $66 * *$ & 6 & -7.636 \\
\hline & & & & & & & & \\
\hline & & & & & & $-27.558 * *$ & & $-22.072 * *$ \\
\hline $\mathrm{P}_{6} \mathrm{xP}_{8}$ & $283 * *$ & $30.451^{* *}$ & $996^{* *}$ & $-11.665 * *$ & $14.468^{* *}$ & 8.654 & $-12.958 * *$ & $-29.624 * *$ \\
\hline $\mathrm{P}_{7} \mathrm{xP}_{8}$ & $15.644^{* *}$ & $-7.599 * *$ & $14.121^{* *}$ & & & $-22.211 * *$ & & $21.723^{* *}$ \\
\hline & & & & & & & & 10.483 \\
\hline & & & & & & & & \\
\hline & & & & & & & -4.66 & \\
\hline
\end{tabular}

*and **: significant at 0.05 and 0.01 levels of probability, respectively

Estimates of heterosis for 100kerenal weight over the mid parent and better parent (Table 8) indicated that 10 and 4 under normal planting date and 15 and 9 crosses under late planting date were significantly posi- tive over MP and $\mathrm{BP}$, respectively it is clear to notice that the $\left(\mathrm{P}_{1} \times \mathrm{P}_{8}\right)$ and $\left(\mathrm{P}_{2} \times \mathrm{P}_{8}\right)$ possessed positive and significant heterosis over MP and BP under both planting dates. This that these crosses could be used source for 
tolerant heat stress in wheat program. Heterosis for 100- kernel weight (Table 8) ranged from $8.228\left(\mathrm{P}_{1} \times \mathrm{P}_{7}\right)$ to $25.765\left(\mathrm{P}_{1} \times \mathrm{P}_{8}\right)$ at $\mathrm{Mp}$ and from 9.456 $\left(\mathrm{P}_{7} \times \mathrm{P}_{8}\right)$ to $10.621 \%\left(\mathrm{P}_{1} \times \mathrm{P}_{8}\right)$ at $\mathrm{BP}$ under normal planting. While, under late planting it ranged from 7.816 $\left(\mathrm{P}_{2} \times \mathrm{P}_{3}\right)$ to $24.77 \%\left(\mathrm{P}_{1} \times \mathrm{P}_{8}\right)$ and from
7.675( $\left(\mathrm{P}_{1} \times \mathrm{P}_{6}\right)$ to $19.946 \%\left(\mathrm{P}_{1} \times \mathrm{P}_{8}\right)$ at MP and BP, respectively (Table 8). These results in agreement with those obtained by, Al-Kodoussi and Hassan (1995), Hassan (1997), Hamada and Twfelis (2001), Nagwa (2007) and Akicnci (2009).

Table (8): Heterosis percentages from mid-parent (MP) and better parent (BP) heterosis for number of kernel/spike and 100- kernel weight of $28 F_{1}$ 's crosses, under recommended $(\mathrm{N})$ and late $(\mathrm{L})$ planting date.

\begin{tabular}{|c|c|c|c|c|c|c|c|c|}
\hline \multirow{3}{*}{$\begin{array}{c}\text { F1 } \\
\text { crosses }\end{array}$} & \multicolumn{4}{|c|}{ Number of kernel / spike } & \multicolumn{4}{|c|}{100 - kernel weight (g) } \\
\hline & \multicolumn{2}{|c|}{$\mathrm{N}$} & \multicolumn{2}{|c|}{$\mathrm{L}$} & \multicolumn{2}{|c|}{$\mathrm{N}$} & \multicolumn{2}{|c|}{$\mathrm{L}$} \\
\hline & MP & $\mathrm{BP}$ & MP & $\mathrm{BP}$ & MP & $\mathrm{BP}$ & MP & $\mathrm{BP}$ \\
\hline $\mathrm{P}_{1} \mathrm{xP}_{2}$ & 4.123 & 2.730 & 5.930 & 0.950 & $11.982 * *$ & $9.651^{* *}$ & $15.057 * *$ & 6.470 \\
\hline $\mathrm{P}_{1} \mathrm{xP}_{3}$ & $11.445 * *$ & & & & 4.375 & & $14.543^{* *}$ & $13.342 * *$ \\
\hline $\mathrm{P}_{1} \mathrm{xP}_{4}$ & $-8.330 * *$ & $19.586^{* *}$ & $-7.847^{*}$ & $15.353 * *$ & 3.208 & & 5.151 & 0.201 \\
\hline $\mathrm{P}_{1} \mathrm{xP}_{5}$ & 1.258 & -3.670 & -4.216 & -6.522 & 3.679 & -2.824 & $12.905^{* *}$ & 5.659 \\
\hline $\mathrm{P}_{1} \mathrm{xP}_{6}$ & 250 & 0.582 & $8.431 * *$ & $8.067^{*}$ & 4.525 & 4.516 & $8.194 *$ & $7.675^{*}$ \\
\hline $\mathrm{P}_{1} \mathrm{xP}_{7}$ & -0.665 & $-10.766^{* *}$ & 1.331 & $-8.987^{*}$ & $8.228 * *$ & 3.387 & $19.481 * *$ & $12.594 * *$ \\
\hline $\mathrm{P}_{1} \mathrm{xP}_{8}$ & -2.157 & $-8.090^{*}$ & -5.230 & $-17.878 * *$ & $25.765^{* *}$ & $10.621 * *$ & $24.777^{* *}$ & $19.946^{* *}$ \\
\hline $\mathrm{P}_{2} \mathrm{XP}_{3}$ & 07 & 097 & $11.237 * *$ & -0.789 & $5.687^{*}$ & -3.861 & $7.816^{*}$ & 0.752 \\
\hline $\mathrm{P}_{2} \mathrm{XP}_{4}$ & 5 & & 1570 & & $6.349^{*}$ & & 7.964* & 4.680 \\
\hline $\mathrm{P}_{2} \mathrm{XP}_{5}$ & & & & & 72 & & -1.217 & $-13.983 * *$ \\
\hline $\mathrm{P}_{2} \mathrm{XP}_{6}$ & & & & & & & & \\
\hline $\mathrm{P}_{2} \mathrm{XP}_{7}$ & & & & & -0.878 & -3. & $4.2 .2 \div$ & $-8.626^{* *}$ \\
\hline $\mathrm{P}_{2} \mathrm{XP}_{8}$ & 236 & 11.6 & 10.10 & $-8.388^{*}$ & $23.328^{* *}$ & $10.512 * *$ & $12.708^{* *}$ & $8.319^{*}$ \\
\hline $\mathrm{P}_{3} \mathrm{xP}_{4}$ & $17.488^{* *}$ & & 17.1 & & $-6.148^{*}$ & $-8.250 * *$ & $16.193^{* *}$ & $11.851^{* *}$ \\
\hline $\mathrm{P}_{3} \mathrm{xP}_{5}$ & 1.989 & & & 2.110 & $-9.084 * *$ & $-10.106^{* *}$ & $8.143^{*}$ & 0.214 \\
\hline $\mathrm{P}_{3} \mathrm{xP}_{6}$ & 452 & $-12.607 * *$ & $7.852 *$ & 0.265 & -0.854 & $-8.039 *$ & $9.437 * *$ & $8.808^{*}$ \\
\hline $\mathrm{P}_{3} \mathrm{XP}_{7}$ & 203 & $18241 * *$ & 2162 & $13.728 * *$ & $-6.200^{*}$ & $-16.591 * *$ & $-11.889^{* *}$ & $-17.785 * *$ \\
\hline $\mathrm{P}_{3} \mathrm{xP}_{8}$ & & & & -1.294 & $11.808^{* *}$ & $-7.813^{*}$ & $16.532 * *$ & $13.172 * *$ \\
\hline $\mathrm{P}_{4} \mathrm{xP}_{5}$ & $92 * *$ & 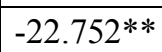 & & $11.455^{* *}$ & $-10.239 * *$ & $-11.264 * *$ & -1.826 & $-12.155^{* *}$ \\
\hline $\mathrm{P}_{4} \mathrm{xP}_{6}$ & & & & & & & & 1.667 \\
\hline $\mathrm{P}_{4} \mathrm{XP}_{7}$ & & & & -13.27 & -1.496 & $-10.611^{* *}$ & $-9.489 * *$ & $-18.476^{* *}$ \\
\hline $\mathrm{P}_{4} \mathrm{xP}_{8}$ & $-6.472 * *$ & $-22.234 * *$ & 2.296 & $-17.465 * *$ & $8.650^{* *}$ & $-8.764 * *$ & $15.900^{* *}$ & $14.849 * *$ \\
\hline $\mathrm{P}_{5} \mathrm{xP}_{6}$ & 4.835 & -4.464 & 5.490 & 2.613 & 2.321 & -4.089 & -5.354 & $-11.824 * *$ \\
\hline $\mathrm{P}_{5} \mathrm{xP}_{7}$ & 0.961 & $-13.220 * *$ & -2.705 & $-14.478 * *$ & $-6.030^{*}$ & $-15.599 * *$ & -5.212 & -5.912 \\
\hline $\mathrm{P}_{5} \mathrm{xP}_{8}$ & 3.780 & 2.404 & $6.930^{*}$ & -5.358 & $11.703^{* *}$ & $-7.067^{*}$ & $9.324 * *$ & -1.381 \\
\hline $\mathrm{P}_{6} \mathrm{xP}_{7}$ & -0.339 & $-6.610^{*}$ & -1.218 & $-11.010^{* *}$ & 4.229 & -0.441 & -0.621 & -6.771 \\
\hline $\mathrm{P}_{6} \mathrm{xP}_{8}$ & & - $>$ & 24.14 & $7.273 *$ & $14.962 * *$ & 1.111 & 5.832 & 2.208 \\
\hline $\mathrm{P}_{7} \mathrm{xP}_{8}$ & & -15. & & \begin{tabular}{|l|}
-13.2 \\
\end{tabular} & $19.379^{* *}$ & $9.456^{* *}$ & $-8.771^{* *}$ & $-17.154^{* *}$ \\
\hline LSD 5\% & & & & & & & & \\
\hline LSD $1 \%$ & & & & 0270 & 7.126 & 8.229 & 8.240 & 9.515 \\
\hline $\begin{array}{l}\text { Average } \\
\text { heterosis }\end{array}$ & 0.676 & -7.701 & 4.449 & -5.091 & 4.574 & -3.311 & 6.396 & 0.505 \\
\hline
\end{tabular}

*and **: significant at 0.05 and 0.01 levels of probability, respectively 


\section{General and specific combining ability effects}

General and specific combining ability effects of the parent, the $28 \mathrm{~F}_{1}$ hybrid and $28 \mathrm{~F}_{2}$-populations for days to heading under the two planting dates are presented in (Table 9). Highly significant negative GCA effects were detected for $\mathrm{P}_{1}, \mathrm{P}_{2}$, $\mathrm{P}_{3}, \mathrm{P}_{6}$ and $\mathrm{P}_{7}$ across environments, indicating that these parents may possess favorable genes which could be utilized in breeding for earliness in wheat. However $\mathrm{P}_{8}$ showed positive and highly significant GCA effects under the tow planting dates, this parent could be considered as a good combiner for lateness.

With regarded to SCA, Eight $F_{1}$ 's and $F_{2}$ crosses showed highly significant negative SCA effects under the two planting date. They could be considered for breeding program for improvement of earliness in wheat. These results are in agreement with those reported by Hendawy (1994), El-Hennawy (1996), ElSayed (1997), El-Sayed et al. (2000) and Akinci (2009).

Concerning the days to maturity, highly significant negative GCA effects for three parents; $\mathrm{P}_{2}, \mathrm{P}_{3}$ and $\mathrm{P}_{7}$ over the two planting dates under $F_{1}$ 's and $F_{2}$ (table 10). This indicts that these parents could decrease days to maturity in their hybrids. These parents may possess favorable genes which be utilized in breeding for ear- liness in wheat. Two parents; $\mathrm{P}_{6}$ and $\mathrm{P}_{8}$ had positive and highly significant over environments for days to maturity, indicating that these parents were considered good combiners to increase for days to maturity under both environments.

With regarded to SCA, three crosses; $\left(\mathrm{P}_{3} \times \mathrm{P}_{5}\right),\left(\mathrm{P}_{3} \times \mathrm{P}_{6}\right)$ and $\left(\mathrm{P}_{4} \times \mathrm{P}_{6}\right)$ had highly significant negative for days to maturity under different environments at $F_{1}$ 's and $F_{2}$ (Table 10 ).

These results are in agreement with those reported by Mann and Sharma (1995) and Zaied (1995).

General and specific combining ability of the parents and SCA effects of the $\mathrm{F}_{1}$ 's hybrids and $\mathrm{F}_{2}$ populations for number of spikes/plant are shown in Table (11). Regarding to GCA for $F_{1}$-crosses and $F_{2}$ population, highly significant and positive GCA effects were detected for two parental genotypes; $\mathrm{P}_{4}$ and $\mathrm{P}_{7}$ under normal planting date and late planting date. Concerning SCA effects for no. of spike/plant proved that $\mathrm{P}_{6} \times \mathrm{P}_{8}$ had highly significant SCA effects in $\mathrm{F}_{1}$ 's and $F_{2}$ under normal planting date. Meanwhile, most of crosses showed no significant and positive SCA in $F_{1}$ 's and $F_{2}$ under the two planting dates; indicating the predominance of non-additive gene effects. These results are in line with those reported by Al-Kodooussi and Hassan (1995), Abdel -kader (2006) and Nagwa (2007). 
Table (9): General (GCA) and specific (SCA) combining ability effects of days to heading.

\begin{tabular}{|c|c|c|c|c|c|c|c|c|c|}
\hline \multirow{2}{*}{ Genotypes } & \multicolumn{2}{|c|}{$\mathbf{F}_{1}$} & \multicolumn{2}{|c|}{$\mathbf{F}_{2}$} & \multirow{2}{*}{ Genotypes } & \multicolumn{2}{|c|}{$\mathbf{F}_{1}$} & \multicolumn{2}{|c|}{$\mathbf{F}_{2}$} \\
\hline & $\mathbf{N}$ & $\mathbf{L}$ & $\mathbf{N}$ & $\mathbf{L}$ & & $\mathbf{N}$ & $\mathbf{L}$ & $\mathbf{N}$ & $\mathbf{L}$ \\
\hline $\mathrm{P}_{1}$ & $-2.208^{* *}$ & $-1.425 * *$ & $-2.467^{* *}$ & $-1.475^{* *}$ & $\mathrm{P}_{2} \mathrm{XP}_{8}$ & $-3.819 * *$ & $-3.007 * *$ & $-1.170^{*}$ & $-3.856^{* *}$ \\
\hline $\mathrm{P}_{2}$ & $-2.375^{* *}$ & $-2.325^{* *}$ & $2.133^{* *}$ & $-2.175^{* *}$ & $\mathrm{P}_{3} \mathrm{xP}_{4}$ & $4.648^{* *}$ & $4.259^{* *}$ & 0.796 & 0.044 \\
\hline $\mathrm{P}_{3}$ & $-0.442 *$ & $-1.892 * *$ & $-1.133^{* * *}$ & $-2.875^{* *}$ & $\mathrm{P}_{3} \mathrm{xP}_{5}$ & -0.052 & 0.493 & $-3.704 * *$ & $-3.089^{* *}$ \\
\hline $\mathrm{P}_{4}$ & 0.192 & 0.308 & $0.967^{* *}$ & $0.858^{* *}$ & $\mathrm{P}_{3} \mathrm{xP}_{6}$ & $-3.185^{* *}$ & $-2.074^{* *}$ & $-4.004^{* *}$ & $-2.422 * *$ \\
\hline $\mathrm{P}_{5}$ & -0.108 & $-0.925 * *$ & $-0.533 * *$ & $-1.342 * *$ & $\mathrm{P}_{3} \mathrm{xP}_{7}$ & 0.515 & -0.041 & $1.163^{* *}$ & $1.944 * *$ \\
\hline $\mathrm{P}_{6}$ & $-0.975^{* *}$ & $-1.358 * *$ & $-1.233 * *$ & $-1.008 * *$ & $\mathrm{P}_{3} \mathrm{xP}_{8}$ & $-3.752 * *$ & $-2.441 * *$ & $-3.504 * *$ & $-2.156^{* *}$ \\
\hline $\mathrm{P}_{7}$ & $-1.675^{* *}$ & $-1.392 * *$ & $-2.400 * *$ & $-0.708 * *$ & $\mathrm{P}_{4} \mathrm{XP}_{5}$ & 0.981 & $1.293 * *$ & $2.196^{* *}$ & $2.178^{* *}$ \\
\hline $\mathrm{P}_{8}$ & $7.592 * *$ & $9.008 * *$ & $8.933 * *$ & $8.725^{* *}$ & $\mathrm{P}_{4} \mathrm{xP}_{6}$ & -0.819 & $3.726^{* *}$ & -0.104 & -0.156 \\
\hline $\mathrm{P}_{1} \mathrm{xP}_{2}$ & $1.981 * *$ & 0.426 & 0.230 & -0.656 & $\mathrm{P}_{4} \mathrm{xP}_{7}$ & -0.119 & -0.241 & $-1.937 * *$ & $2.878^{* *}$ \\
\hline $\mathrm{P}_{1} \mathrm{xP}_{3}$ & 1.048 & $0.993 *$ & $-3.437^{* *}$ & $-2.956 * *$ & $\mathrm{P}_{4} \mathrm{XP}_{8}$ & $-3.385^{* *}$ & $-5.641^{* *}$ & $-5.270 * *$ & $-6.222 * *$ \\
\hline $\mathrm{P}_{1} \mathrm{XP}_{4}$ & $-2.585^{* *}$ & $-4.207 * *$ & $3.130^{* *}$ & -0.689 & $\mathrm{P}_{5} \mathrm{xP}_{6}$ & $2.148^{* *}$ & $2.959 * *$ & -0.604 & $2.711^{* *}$ \\
\hline $\mathrm{P}_{1} \mathrm{xP}_{5}$ & -0.619 & $1.026^{*}$ & $1.630 * *$ & 0.511 & $\mathrm{P}_{5} \mathrm{xP}_{7}$ & $2.181 * *$ & 0.326 & 0.563 & 0.411 \\
\hline $\mathrm{P}_{1} \mathrm{xP}_{6}$ & 0.915 & $-1.541 * *$ & $-0.670^{* *}$ & -0.822 & $\mathrm{P}_{5} \mathrm{xP}_{8}$ & $-2.085^{* *}$ & $-3.407 * *$ & $-3.770 * *$ & $-2.689^{* *}$ \\
\hline $\mathrm{P}_{1} \mathrm{xP}_{7}$ & $3.615^{* *}$ & 4.15 & 1.170 & $3.878 * *$ & $\mathrm{P}_{6} \mathrm{xP}_{7}$ & ** & $-4.574 * *$ & -0.737 & 1.744 \\
\hline $\mathrm{P} 1 \mathrm{xP}_{8}$ & $-1.985^{* *}$ & $-3.907 * *$ & $-2.837^{* *}$ & $-2.556 * *$ & $\mathrm{P}_{6} \mathrm{xP}_{8}$ & $-3.219^{* *}$ & $-3.974 * *$ & $-4.070^{* *}$ & $-4.356^{* *}$ \\
\hline $\mathrm{P}_{2} \mathrm{xP}_{3}$ & $4.215^{* *}$ & $1.893^{* *}$ & $8.896 * *$ & $7.744 * *$ & $\mathrm{P}_{7} \mathrm{KP}_{8}$ & $-4.185^{* *}$ & $-2.941^{* *}$ & -0.904 & $-4.656^{* *}$ \\
\hline $\mathrm{P}_{2} \mathrm{XP}_{4}$ & $-2.752 * *$ & $-3.307 * *$ & $-4.204 * *$ & 0.011 & gi & .0 .190 & 0.159 & 0.162 & 0.226 \\
\hline $\mathrm{P}_{2} \mathrm{XP}_{5}$ & 0.215 & 0.259 & 0.296 & 1.211 & S.E. sij & 0.583 & 0.488 & 0.496 & 0.692 \\
\hline $\mathrm{P}_{2} \mathrm{XP}_{6}$ & $2.415^{* *}$ & $-1.307 * *$ & -0.004 & $-2.122 * *$ & S.E. (gi-gj) & 0.288 & 0.241 & 0.245 & 0.341 \\
\hline $\mathrm{P}_{2} \mathrm{xP}_{7}$ & $3.148^{* *}$ & $4.393 * *$ & $-1.837^{* *}$ & $-2.422 * *$ & S.E. (sij-sik) & 0.863 & 0.722 & 0.734 & 1.024 \\
\hline
\end{tabular}

* and **: significant at 0.05 and 0.01 levels of probability, respectively 
Table (10): General (GCA) and specific (SCA) combining ability effects of Days to maturity.

\begin{tabular}{|c|c|c|c|c|c|c|c|c|c|}
\hline \multirow{2}{*}{ Genotypes } & \multicolumn{2}{|c|}{$F_{1}$} & \multicolumn{2}{|c|}{$\mathbf{F}_{2}$} & \multirow{2}{*}{ Genotypes } & \multicolumn{2}{|c|}{$F_{1}$} & \multicolumn{2}{|c|}{$F_{2}$} \\
\hline & $\mathbf{N}$ & $\mathbf{L}$ & $\mathbf{N}$ & $\mathbf{L}$ & & $\mathbf{N}$ & $\mathbf{L}$ & $\mathbf{N}$ & $\mathbf{L}$ \\
\hline $\mathrm{P}_{1}$ & $0.483 * *$ & $-0.525 * *$ & $0.608 * *$ & $-0.392 *$ & $I_{2} \times \Gamma_{8}$ & 0.733 & 48 & $.252 * *$ & $-4.041 * *$ \\
\hline $\mathrm{P}_{2}$ & $383 * *$ & $-2.125^{* *}$ & $-1.358 * *$ & $-2.758 * *$ & $\mathrm{P}$ yP & $2.267 * *$ & -0.319 & $-2.381 * *$ & $-2.707 * *$ \\
\hline $\mathrm{P}_{3}$ & $*$ & * & $* *$ & - & $\mathrm{P}_{3} \mathrm{xP}_{5}$ & $* *$. & $* *$ & $* *$ & \\
\hline $\mathrm{P}_{4}$ & $50 * *$ & -0.058 & $-1.158 * *$ & .30 & $\mathrm{P}_{3} \mathrm{xP}_{6}$ & $-1.267 *$ & $-1.552 * *$ & -2.4 & $-5.807 * *$ \\
\hline $\mathrm{P}_{5}$ & $17 * *$ & $0.842 * *$ & -0.125 & 1.07 & $\mathrm{P}_{3} \mathrm{xP}_{7}$ & -0.033 & -0.852 & $-3.848 * *$ & $-4.874 * *$ \\
\hline $\mathrm{P}_{6}$ & $* *$ & 0 & $* *$ & 0 & $13 \times 8$ & $0 * *$ & $* *$ & $*$ & 2 \\
\hline $\mathrm{P}_{7}$ & $83 * *$ & $-3.192 * *$ & $-2.692 * *$ & $-2.525 * *$ & $\mathrm{P}_{4} \mathrm{xP}_{5}$ & $-2.100 * *$ & $-1.219 *$ & $2.219 * *$ & -0.041 \\
\hline $\mathrm{P}_{8}$ & $4.317 * *$ & $5.942 * *$ & $5.375^{* *}$ & $6.808 * *$ & $\mathrm{P}_{4} \mathrm{xP}_{6}$ & $-5.533 * *$ & $-2.219 * *$ & $-1.781 * *$ & $1.293^{*}$ \\
\hline $\mathrm{P}_{1} \mathrm{xP}_{2}$ & 0.567 & 1 & 00 & 01 & that & $* *$. & -5.1 & -4. & -0 . \\
\hline $\mathrm{P}_{1} \mathrm{XP}_{3}$ & 133 & 0.481 & 185 & $-3.674 * *$ & $\mathrm{P}_{4} \mathrm{XP}_{8}$ & $2.100^{* *}$ & -0.319 & $-7.281 * *$ & $1.559^{* *}$ \\
\hline $\mathrm{P}_{1} \mathrm{xP}_{4}$ & $1.933 * *$ & $5.148 * *$ & 0.485 & 0.759 & $\mathrm{P}_{5} \mathrm{xP}_{6}$ & -0.633 & $5.548 * *$ & $-5.815^{* *}$ & -0.141 \\
\hline $\mathrm{P}_{1} \mathrm{xP}_{5}$ & -0.833 & $752 * *$ & -0.215 & 5 & $\mathrm{P}_{5} \mathrm{xP}_{7}$ & $*$ & -( & ** & 0 \\
\hline $\mathrm{P}_{1} \mathrm{xP}_{6}$ & $2.733 * *$ & $1.248^{*}$ & $1.452 * *$ & 2.32 & $\mathrm{P}_{5} \mathrm{xP}_{8}$ & -0.0 & -2.2 & 0.685 & -0.541 \\
\hline $\mathrm{P}_{1} \mathrm{XP}_{7}$ & $2.967 * *$ & $4.281 * *$ & $4.685^{* *}$ & $3.593 * *$ & $\mathrm{P}_{6} \mathrm{xP}_{7}$ & $-2.500 * *$ & $-1.419^{*}$ & -0.248 & -0.874 \\
\hline $\mathrm{P} 1 \mathrm{xP}_{8}$ & 367 & 148 & .048 & $2.259 *$ & $\mathrm{P}_{6} \mathrm{xP}_{8}$ & -0.433 & $-2.219 * *$ & $-1.315^{*}$ & -2.8 \\
\hline $\mathrm{P}_{2} \mathrm{xP}_{3}$ & -0.100 & $3.415^{* *}$ & 0.819 & $8.693 * *$ & $\mathrm{P}_{7} \mathrm{xP}_{8}$ & $1.133^{*}$ & 0.148 & $4.252 * *$ & -0.607 \\
\hline $\mathrm{P}_{2} \mathrm{xP}_{4}$ & 0.300 & 0.748 & 0.452 & $-2.874 * *$ & S.E. gi & 0.018 & 0.195 & 0.180 & 0.193 \\
\hline $\mathrm{P}_{2} \mathrm{XP}_{5}$ & $-1.800 * *$ & $-2.152 * *$ & $2.419 * *$ & $-6.641 * *$ & S.E. sij & 0.553 & 0.597 & 0.551 & 0.592 \\
\hline $\mathrm{P}_{2} \mathrm{xP}_{6}$ & $1.100^{*}$ & $-3.152 * *$ & -0.248 & $1.693 * *$ & S.E. (gi-gj) & 0.562 & 0.491 & 0.272 & 0.292 \\
\hline $\mathrm{P}_{2} \mathrm{xP}_{7}$ & -0.667 & -0.452 & $-2.015 * *$ & 0.959 & S.E. (sij-sik) & 0.818 & 0.884 & 0.816 & 0.876 \\
\hline
\end{tabular}

* and **: significant at 0.05 and 0.01 levels of probability, respectively 
Table (11): General (GCA) and specific (SCA) combining ability effects of no. of spike / plant .

\begin{tabular}{|c|c|c|c|c|c|c|c|c|c|}
\hline \multirow{2}{*}{ Genotypes } & \multicolumn{2}{|c|}{$F_{1}$} & \multicolumn{2}{|c|}{$\mathbf{F}_{2}$} & \multirow{2}{*}{ Genotypes } & \multicolumn{2}{|c|}{$\mathbf{F}_{1}$} & \multicolumn{2}{|c|}{$\mathbf{F}_{2}$} \\
\hline & $\mathbf{N}$ & $\mathbf{L}$ & $\mathbf{N}$ & $\mathbf{L}$ & & $\mathbf{N}$ & $\mathbf{L}$ & $\mathbf{N}$ & $\mathbf{L}$ \\
\hline $\mathrm{P}_{1}$ & $318 *$ & -0.187 & -0.269 & -0.077 & $\mathrm{P}_{2} \mathrm{XP}_{8}$ & 0.265 & 0.026 & -0.140 & -0.070 \\
\hline $\mathrm{P}_{2}$ & $0.676^{* *}$ & -0.032 & $0.393 *$ & $-0.235^{*}$ & $\mathrm{P}_{3} \mathrm{xP}_{4}$ & 0.551 & 0.256 & 0.541 & 0.032 \\
\hline $\mathrm{P}_{3}$ & $684 * *$ & $-0.265^{*}$ & $-0.649 * *$ & $-0.208^{*}$ & $\mathrm{P}_{3} \mathrm{XP}_{5}$ & 0.535 & 0.386 & 0.503 & 0.554 \\
\hline $\mathrm{P}_{4}$ & $1.039 * *$ & $0.452 * *$ & $1.123 * *$ & $0.353^{* *}$ & $\mathrm{P}_{3} \mathrm{xP}_{6}$ & 0.658 & $0.716^{*}$ & 0.334 & $0.671^{*}$ \\
\hline $\mathrm{P}_{5}$ & $-0.344 *$ & -0.045 & $-0.513 * *$ & 0.018 & $\mathrm{P}_{3} \mathrm{xP}_{7}$ & -0.709 & 0.246 & -0.384 & 0.058 \\
\hline $\mathrm{P}_{6}$ & $68 * *$ & $-0.308 * *$ & $-0.977 * *$ & -0.23 & $13 \Lambda$ & -0.009 & 0.159 & -0.718 & 0.222 \\
\hline $\mathrm{P}_{7}$ & $1.066^{* *}$ & $0.228 *$ & $1.177 * *$ & $0.247^{* *}$ & $\mathrm{P}_{4} \mathrm{xP}_{5}$ & $-2.155^{* *}$ & -0.064 & $-2.176^{* *}$ & 0.100 \\
\hline $\mathrm{P}_{8}$ & -0.168 & 0.184 & -0.203 & 0.143 & $\mathrm{P}_{4} \mathrm{xP}_{6}$ & k* & 0.066 & *** & 0.110 \\
\hline $\mathrm{P}_{1} \mathrm{xP}_{2}$ & 0.081 & -0.047 & -0.889 & -0.024 & $\mathrm{P}_{4} \mathrm{xP}_{7}$ & 02 & -0.171 & 0.207 & -0.116 \\
\hline $\mathrm{P}_{1} \mathrm{xP}_{3}$ & -0.625 & 0.619 & -0.780 & $0.556^{*}$ & $\mathrm{P}_{4} \mathrm{xP}_{8}$ & 0.035 & -0.257 & -0.297 & -0.292 \\
\hline $\mathrm{P}_{1} \mathrm{xP}_{4}$ & 0.418 & -0.097 & 0.294 & 0.035 & $\mathrm{P}_{5} \mathrm{xP}_{6}$ & 0.185 & 0.496 & 0.024 & 0.478 \\
\hline $\mathrm{P}_{1} \mathrm{XP}_{5}$ & 0.268 & 0.433 & 0.076 & 0.383 & $\mathrm{P}_{5} \mathrm{XP}_{7}$ & 0185 & -0.007 & -0.940 & -0.034 \\
\hline $\mathrm{P}_{1} \mathrm{xP}_{6}$ & 0.125 & 0.463 & 0.333 & $0.667 *$ & $\mathrm{P}_{5} \mathrm{xP}_{8}$ & 0.018 & $0.806^{*}$ & 0.039 & 0.756 \\
\hline $\mathrm{P}_{1} \times \mathrm{P}_{7}$ & 25 & 0107 & 0100 & -0.1 & $\mathrm{P}_{6} \mathrm{XP}_{7}$ & 0 & * & 0.900 & 0.930 \\
\hline $\mathrm{P} 1 \mathrm{xP}_{8}$ & -0.175 & -0.261 & -0.045 & -0.355 & $\mathrm{P}_{6} \mathrm{xP}_{8}$ & $1.275^{* *}$ & 0.269 & $1.149^{* *}$ & 0.100 \\
\hline $\mathrm{P}_{2} \mathrm{XP}_{3}$ & -0.552 & -0.194 & -0.829 & 0.027 & $\mathrm{P}_{7} \mathrm{xP}_{8}$ & -0.092 & -0.367 & -0.465 & -0.306 \\
\hline $\mathrm{P}_{2} \mathrm{XP}_{4}$ & $1.525^{* *}$ & 0.589 & 0.925 & -0.240 & S.E. gi & 0.142 & 113.000 & 0.019 & 0.093 \\
\hline $\mathrm{P}_{2} \mathrm{XP}_{5}$ & 0.175 & 0.086 & -0.312 & -0.146 & S.E. sij & 0.434 & 0.347 & 0.575 & 0.284 \\
\hline $\mathrm{P}_{2} \mathrm{XP}_{6}$ & -0.569 & 0.583 & -0.57 & .505 & S.E. (gi-gj) & 214 & 0.171 & 0.284 & 0.140 \\
\hline $\mathrm{P}_{2} \mathrm{xP}_{7}$ & -0.035 & -0.054 & 0.257 & -0.174 & S.E. (sij-sik) & 0.647 & 0.347 & 0.851 & 0.396 \\
\hline
\end{tabular}

* and **: significant at 0.05 and 0.01 levels of probability, respectively 
General combining ability effects for grain yield/plant were positive and highly significant for $\mathrm{P}_{4}$ and $\mathrm{P}_{7}$ under normal and late planting date in $F_{1}$ 's and $F_{2}$ (Table 12). It could be concluded that $\mathrm{P}_{4}$ and $\mathrm{P}_{7}$ were considered good combiners for grain yield under both planting dates. With respect to SCA, some crosses showed positive and no significant for grain yield/plant under normal planting and late date.

These results were in line with those reported by Bakheit et al. (1989), Al-Kodooussi and Hassan (1991), Abdel -kader (2006), Mohamed (2007) and Akinci (2009).

General combining ability effects of $F_{1}$ 's and $F_{2}$ were positive and highly significant for $\mathrm{P}_{4}$ and $\mathrm{P}_{7}$ under both normal and late planting date (Table13). Therefore, they could be considered good combiners for biological yield. The best hybrids displayed positive and significant or highly significant SCA effects of $F_{1}$ 's for biological yield were $\mathrm{P}_{1} \times \mathrm{P}_{4}$, $\mathrm{P}_{1} \times \mathrm{P}_{6}, \quad \mathrm{P}_{2} \times \mathrm{P}_{7}, \mathrm{P}_{4} \times \mathrm{P}_{7}$ and $\mathrm{P}_{6} \times \mathrm{P}_{8}$, under normal planting date. While, the hybrid $\mathrm{P}_{2} \times \mathrm{P}_{4}$ showed significant and positive SCA affects under late planting date. SCA effects of $F_{2}$ population showed that $\mathrm{P}_{2} \times \mathrm{P}_{6}$ and $\mathrm{P}_{4} \times \mathrm{P}_{8}$ had positive and significant SCA effects under late planting date. Most population has negative effects under normal planting date. These results were agree with those reported by Zaied (1995), Tawfelis et al. (2006).and Barhim and Mohamed (2014).

General and specific combining ability effects of the parents of the $\mathrm{F}_{1}$ hybrid and $\mathrm{F}_{2}$-populations for number of Kernel/spike are presented in Table (14). General combining ability effects of $F_{1}$ 's and $F_{2}$ parents had positive significantly for $\mathrm{P}_{2}, \mathrm{P}_{4}$ and $\mathrm{P}_{7}$ under normal and late planting date. Therefore, they could be considered good combiners for number of kernel/spike. The best hybrid was $\mathrm{P}_{3} \times \mathrm{P}_{4}$ had positive and highly significant SCA effects of both $F_{1}$ 's and $F_{2}$ populations for number of Kernel/spike under both planting dates. These results are in line with those obtained by El-Borhamy (1995), Zaied (1995), El-Henawy (1996), Abdel -kader (2006), El-Sayed (1997), El-Sayed et al. (2000) and El-Karamity et al. (2007).

General combining ability effects of both $\mathrm{F}_{1}$ 's and $\mathrm{F}_{2}$ generations were positive and highly significant for $\mathrm{P}_{3}$ and $\mathrm{P}_{5}$ under normal and late planting date (table 15). These could be consider good combiner, for number of 100-Kernel weight across environments. Regarding $\mathrm{F}_{1}$ 's and $\mathrm{F}_{2}$ SCA, only one hybrid; showed $\left(\mathrm{P}_{3} \times \mathrm{P}_{8}\right)$ significantly positive under normal and late planting dates, for 100 -Kernel weight (Table 13).

Similar results obtained by $\mathrm{Zu}-$ bair et al. (1987), El-Shami et al. (1996), El-Sayed (1997), El-Sayed et al. (2000) and Nagwa (2007). 
Table (12): General (GCA) and specific (SCA) combining ability effects of Grain yield / plant (g).

\begin{tabular}{|c|c|c|c|c|c|c|c|c|c|}
\hline \multirow{2}{*}{ Genotypes } & \multicolumn{2}{|c|}{$\mathbf{F}_{1}$} & \multicolumn{2}{|c|}{$F_{2}$} & \multirow{2}{*}{ Genotypes } & \multicolumn{2}{|c|}{$\mathbf{F}_{1}$} & \multicolumn{2}{|c|}{$F_{2}$} \\
\hline & $\mathbf{N}$ & $\mathbf{L}$ & $\mathbf{N}$ & $\mathbf{L}$ & & $\mathbf{N}$ & $\mathbf{L}$ & $\mathbf{N}$ & $\mathbf{L}$ \\
\hline $\mathrm{P}_{1}$ & 0.424 & 0.367 & $-1.501 * *$ & 0.183 & & 0.416 & -0.331 & -1.144 & $-1.891^{*}$ \\
\hline $\mathrm{P}_{2}$ & $1.720 * *$ & $1.145^{* *}$ & $1.080^{* *}$ & 0.417 & $\mathrm{P}_{3} \mathrm{xP}_{4}$ & -1.551 & 1.665 & $-3.383 * *$ & -0.192 \\
\hline $\mathrm{P}_{3}$ & $-2.211 * *$ & $-1.532 * *$ & $-1.295 * *$ & $-1.046^{* *}$ & $\mathrm{P}_{3} \mathrm{xP}_{5}$ & 1.207 & -0.690 & -0.327 & 0.542 \\
\hline $\mathrm{P}_{4}$ & $2.505^{* *}$ & $2.285 * *$ & $1.701 * *$ & $1.889 * *$ & $\mathrm{P}_{3} \mathrm{xP}_{6}$ & 1.867 & 0.410 & 0.711 & 1.516 \\
\hline $\mathrm{P}_{5}$ & $-1.130 * *$ & $-0.810^{*}$ & -0.039 & -0.372 & - & 86 & 1.150 & -3.1 & -0.783 \\
\hline $\mathrm{P}_{6}$ & $-2.254 * *$ & $-2.737 * *$ & $-0.753 * *$ & $-1.739 * *$ & $\mathrm{P}_{3} \mathrm{xP}_{8}$ & 0.337 & -0.114 & $2.361^{*}$ & -0.314 \\
\hline $\mathrm{P}_{7}$ & $2.859^{* *}$ & $2.059 * *$ & $1.685^{* *}$ & $1.834 * *$ & $\mathrm{P}_{4} \mathrm{xP}_{5}$ & $-3.229^{*}$ & -1.880 & $-3.172 * *$ & -1.760 \\
\hline $\mathrm{P}_{8}$ & $-1.913 * *$ & $-0.777 *$ & $-0.877 * *$ & $-1.168^{* *}$ & $\mathrm{P}_{4} \times \mathrm{P}_{6}$ & -2.018 & -1.406 & -1.305 & $-2.089 *$ \\
\hline $\mathrm{P}_{1} \mathrm{xP}_{2}$ & 0.572 & -0.704 & $-3.216^{* *}$ & -0.833 & $\mathrm{P}_{4} \mathrm{xP}_{7}$ & $2.499 *$ & 1.074 & -0.243 & 0.631 \\
\hline $\mathrm{P}_{1} \mathrm{xP}_{3}$ & 1 & -0.961 & 0.822 & -0.676 & $\mathrm{P}_{4} \times \mathrm{P}_{8}$ & -2.1 & -1.234 & -1.9 & 1.501 \\
\hline $\mathrm{P}_{1} \mathrm{xP}_{4}$ & $3.051^{* *}$ & -0.037 & $-2.277 * *$ & -0.988 & $\mathrm{P}_{5} \mathrm{xP}_{6}$ & 0.490 & 1.156 & -0.005 & 0.725 \\
\hline $\mathrm{P}_{1} \mathrm{XP}_{5}$ & 976 & -0.585 & $-2.017^{*}$ & 0.033 & $\mathrm{P}_{5} \mathrm{xP}_{7}$ & 1.187 & 0.126 & $-2.963 * *$ & -1.008 \\
\hline $\mathrm{P}_{1} \mathrm{xP}_{6}$ & $4.736^{* *}$ & 0.242 & 1.077 & 0.567 & $\mathrm{P}_{5} \mathrm{xP}_{8}$ & -0.341 & -0.592 & 1.292 & -0.022 \\
\hline $\mathrm{P}_{1} \mathrm{xP}_{7}$ & 0.700 & -0.742 & -1.691 & -0.173 & $\mathrm{P}_{6} \mathrm{xP}_{7}$ & $-2.786^{*}$ & -0.477 & 0.281 & 0.395 \\
\hline $\mathrm{P} 1 \mathrm{xP}_{8}$ & 0.256 & 1.428 & -1.623 & $-2.633 * *$ & $\mathrm{P}_{6} \mathrm{xP}_{8}$ & $3.043 * *$ & 0.409 & -0.217 & -0.071 \\
\hline $\mathrm{P}_{2} \mathrm{XP}_{3}$ & 1.417 & -1.722 & 1210 & -1.020 & $\mathrm{P}_{7} \mathrm{XP}_{8}$ & 1123 & 1.052 & -1.426 & -0.127 \\
\hline $\mathrm{P}_{2} \mathrm{XP}_{4}$ & 1.934 & $2.651^{* *}$ & -1.888 & -0.462 & S.E. gi & 0.371 & 0.325 & 0.289 & 0.313 \\
\hline $\mathrm{P}_{2} \mathrm{XP}_{5}$ & -2.008 & -0.804 & -0.631 & $-2.634^{* *}$ & S.E. sij & 1.139 & 0.996 & 0.885 & 0.960 \\
\hline $\mathrm{P}_{2} \mathrm{XP}_{6}$ & -2.091 & 0.497 & -0.667 & 1.170 & S.E. (gi-gj) & 0.562 & 0.491 & 0.436 & 0.474 \\
\hline $\mathrm{P}_{2} \mathrm{xP}_{7}$ & 1.870 & 0.327 & -1.123 & 0.063 & S.E. (sij-sik) & 1.685 & 1.474 & 1.309 & 1.421 \\
\hline
\end{tabular}

* and **: significant at 0.05 and 0.01 levels of probability, respectively 
Table (13): General (GCA) and specific (SCA) combining ability effects of biological yield / plant(g).

\begin{tabular}{|c|c|c|c|c|c|c|c|c|c|}
\hline \multirow{2}{*}{ Genotypes } & \multicolumn{2}{|c|}{$\mathbf{F}_{1}$} & \multicolumn{2}{|c|}{$\mathbf{F}_{2}$} & \multirow{2}{*}{ Genotypes } & \multicolumn{2}{|l|}{$\mathbf{F}_{1}$} & \multicolumn{2}{|c|}{$F_{2}$} \\
\hline & $\mathbf{N}$ & $\mathbf{L}$ & $\mathbf{N}$ & $\mathbf{L}$ & & $\mathbf{N}$ & $\mathbf{L}$ & $\mathbf{N}$ & $\mathbf{L}$ \\
\hline $\mathrm{P}_{1}$ & 0 . & & $*$ & 1 & & 1 & 21 & .661 & r \\
\hline $\mathrm{P}_{2}$ & -5 & & & 1. & & & 276 & & 2.610 \\
\hline $\mathrm{P}_{3}$ & $-6.890 * *$ & $8 * *$ & $* *$ & - & & 1485 & 190 & -1.872 & .584 \\
\hline $\mathrm{P}_{4}$ & $*$ & $2 * *$ & $* *$ & 6.109 & $\mathrm{xP}$ & 859 & 203 & 0.196 & 999.000 \\
\hline $\mathrm{P}_{5}$ & 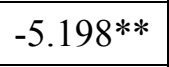 & $-3.469 * *$ & -0 & ** & & 72 & 0.729 & -9.29 & -3.057 \\
\hline $\mathrm{P}_{6}$ & 0.012 & $-7.155 * *$ & $-2.348 * *$ & (2) & $\mathrm{xP}_{8}$ & 0.847 & -0.784 & 1.1. & -1.068 \\
\hline $\mathrm{P}_{7}$ & $* *$ & $\% *$ & $70 * *$ & $4.993 * *$ & $\mathrm{P}_{4} \mathrm{xP}_{5}$ & $52 *$ & -4.893 & $-9.618 * *$ & $-7.252 * *$ \\
\hline $\mathrm{P}_{8}$ & $-3.340 * *$ & -0.451 & -0.550 & -8 & $\mathrm{P}_{4} \mathrm{xP}_{6}$ & $* *$ & -4.547 & -6.8 & $-7.947^{*}$ \\
\hline $\mathrm{P}_{1} \mathrm{xP}_{2}$ & $-6.618^{*}$ & & 1 & ( & & $8 * *$ & 6 & 0.722 & 3 \\
\hline $\mathrm{P}_{1} \mathrm{XP}_{3}$ & .776 & -5.550 & 5J5 & -2.104 & $\mathrm{P}_{4} \mathrm{XP}_{8}$ & $-10.46^{* *}$ & -4.554 & $-7.608 * *$ & $5.602 *$ \\
\hline $\mathrm{P}_{1} \mathrm{xP}_{4}$ & $077 * *$ & 341 & $-8.833 * *$ & -3.774 & $\mathrm{P}_{5} \mathrm{xP}_{6}$ & 221 & 1.461 & -1.606 & 1.494 \\
\hline $\mathrm{P}_{1} \mathrm{xP}_{5}$ & 105 & & 40 & 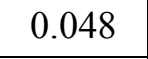 & $\mathrm{P}_{5} \mathrm{xP}_{7}$ & & 1.937 & -8.66 & -2.709 \\
\hline $\mathrm{P}_{1} \mathrm{xP}_{6}$ & $11.899 * *$ & -0.577 & 0.039 & 0.089 & & 569 & -3.603 & 0.929 & -0.740 \\
\hline $\mathrm{P}_{1} \mathrm{xP}_{7}$ & 955 & -0.447 & $-7.179 * *$ & -0.597 & $\mathrm{P}_{6} \mathrm{xP}_{7}$ & -2.566 & -0.256 & -3.333 & -0.661 \\
\hline $\mathrm{P} 1 \mathrm{xP}_{8}$ & & & & $.978 * *$ & & $6.310^{*}$ & -1.930 & 0.15 & 32 \\
\hline $\mathrm{P}_{2} \mathrm{xP}_{3}$ & 2.565 & -5.921 & -3.415 & -3.876 & $\mathrm{P}_{7} \mathrm{xP}_{8}$ & -1.361 & 4.106 & $-5.438^{*}$ & 4.175 \\
\hline $\mathrm{P}_{2} \mathrm{XP}_{4}$ & 1.822 & $7.986^{*}$ & $-9.868 * *$ & -1.090 & & 1.051 & 1.100 & 0.743 & 0.850 \\
\hline $\mathrm{P}_{2} \mathrm{XP}_{5}$ & $-7.030^{*}$ & -1.270 & -4.054 & $-5.915^{*}$ & & 3.220 & 3.371 & 2.277 & 2.605 \\
\hline $\mathrm{P}_{2} \mathrm{XP}_{6}$ & -4.806 & -0.903 & & $5.200 *$ & F.E. (gi-gj) & 1.588 & 1.663 & 1.123 & 1.285 \\
\hline $\mathrm{P}_{2} \mathrm{XP}_{7}$ & $9.217 * *$ & -0.317 & -2.031 & -0.856 & S.E. (sij-sik) & 4.765 & 4.988 & 3.369 & 3.854 \\
\hline
\end{tabular}

* and **: significant at 0.05 and 0.01 levels of probability, respectively 
Table (14): General (GCA) and specific (SCA) combining ability effects of number kernel / plant.

\begin{tabular}{|c|c|c|c|c|c|c|c|c|c|}
\hline \multirow{2}{*}{ Genotypes } & \multicolumn{2}{|c|}{$\mathbf{F}_{1}$} & \multicolumn{2}{|c|}{$\mathbf{F}_{2}$} & \multirow{2}{*}{ enotypes } & \multicolumn{2}{|c|}{$F_{1}$} & \multicolumn{2}{|c|}{$\mathbf{F}_{2}$} \\
\hline & $\mathbf{N}$ & $\mathbf{L}$ & $\mathbf{N}$ & $\mathbf{L}$ & & $\mathbf{N}$ & $\mathbf{L}$ & $\mathbf{N}$ & $\mathbf{L}$ \\
\hline$x_{1}$ & $670^{*}$ & & -1.122 & & & -0.814 & 0.723 & -1.806 & .093 \\
\hline $\mathrm{P}_{2}$ & & $*$ & & 3 & $\mathrm{P}_{3} \mathrm{xP}_{4}$ & $*$ & $*$ & $*$ & 0.579 \\
\hline $\mathrm{P}_{3}$ & $*$ & $-2.385 * *$ & $-3.743 * *$ & -2.41 & & -0.846 & $0.5 / 1$ & 0.309 & $-4.387^{*}$ \\
\hline $\mathrm{P}_{4}$ & & $2.667 * *$ & $3.851^{* *}$ & 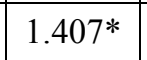 & $\mathrm{P}_{3} \mathrm{xP}_{6}$ & & 0.000 & 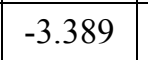 & 1.850 \\
\hline $\mathrm{P}_{5}$ & & $* *$ & $* *$. & -2.4 & 2 & $-4.508^{*}$ & 2 & $*$ & 0.232 \\
\hline $\mathrm{P}_{6}$ & 776 & 0.942 & $.713 *$ & 0.99 & $\mathrm{P}_{3} \mathrm{xP}_{8}$ & -0.387 & -1.633 & $-6.404 * *$ & 0.315 \\
\hline $\mathrm{P}_{7}$ & $101 * *$ & $5.057 * *$ & $4.750 * *$ & $5.745^{* *}$ & $\mathrm{P}_{4} \mathrm{xP}_{5}$ & -4.3 & 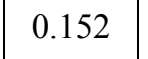 & -2 . & -2.774 \\
\hline $\mathrm{P}_{8}$ & $*$ & $-6.769 * *$ & $-5.535 * *$ & $-6.935^{* *}$ & $\mathrm{P}_{4} \mathrm{XP}_{6}$ & ** & $96 *$ & -10 & 1.544 \\
\hline $\mathrm{P}_{1} \mathrm{xP}_{2}$ & & 1237 & 2.181 & 0.413 & $\mathrm{P}_{\mathrm{P}}^{\mathrm{P}_{3}}$ & -0.759 & $-5.288 * *$ & $-7.519 * *$ & $-7.072 * *$ \\
\hline $\mathrm{P}_{1} \mathrm{xP}_{3}$ & $4.643 *$ & 250 & $3073 * *$ & 3402 & $\mathrm{P}_{4} \mathrm{xP}_{8}$ & 2501 & 0742 & -2.768 & $-4.712^{*}$ \\
\hline $\mathrm{P}_{1} \mathrm{xP}_{4}$ & 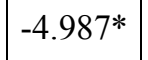 & 0 & $1 * *$ & -1.349 & $\mathrm{P}_{5} \mathrm{xP}_{6}$ & 2.005 & 0.484 & $0.0 / 2$ & 1.297 \\
\hline $\mathrm{P}_{1} \mathrm{XP}_{5}$ & -0.024 & -2.629 & -2.286 & -3.575 & $\mathrm{P}_{5} \mathrm{xP}_{7}$ & 1.105 & -1.328 & 0.549 & -1.465 \\
\hline $\mathrm{P}_{1} \mathrm{xP}_{6}$ & 3 & 2.917 & 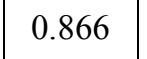 & ent & $\mathrm{P}_{5} \mathrm{xP}_{8}$ & & 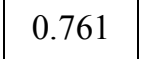 & . & 2.8 \\
\hline $\mathrm{P}_{1} \mathrm{xP}_{7}$ & -0.239 & 1.885 & -0.307 & -1.050 & $\mathrm{P}_{6} \mathrm{xP}_{7}$ & 0.581 & -1.956 & -0.475 & -0.855 \\
\hline $\mathrm{P} 1 \mathrm{xP}_{8}$ & -1.471 & -4.386 & $-3.649 *$ & -2.253 & $\mathrm{P}_{6} \mathrm{xP}_{8}$ & 515 & $7.753 * *$ & $5.866^{* *}$ & -2.315 \\
\hline $\mathrm{P}_{2} \mathrm{XP}_{3}$ & 0.201 & 1.060 & 0.072 & -0.305 & $\mathrm{P}_{7} \mathrm{XP}_{8}$ & 4 & 4138 & 1.610 & 1.130 \\
\hline $\mathrm{P}_{2} \mathrm{xP}_{4}$ & 1.103 & -1.959 & -3.345 & -3.612 & & .688 & 0.732 & 0.598 & 0.638 \\
\hline $\mathrm{P}_{2} \mathrm{XP}_{5}$ & 0.200 & 4.184 & -0.237 & -0.665 & S.E. sij & 2.110 & 2.245 & 1.835 & 1.955 \\
\hline $\mathrm{P}_{2} \mathrm{xP}_{6}$ & 2.843 & $4.939 *$ & 1.586 & 1.625 & S.E. (gi-gj) & 1.041 & 1.107 & 0.905 & 0.964 \\
\hline $\mathrm{P}_{2} \mathrm{XP}_{7}$ & 0.485 & -0.316 & -2.564 & -0.470 & S.E. (sij-sik) & 3.122 & 3.321 & 2.715 & 2.893 \\
\hline
\end{tabular}

*and **: significant at 0.05 and 0.01 levels of probability, respectively 
Table (15): General (GCA) and specific (SCA) combining ability effects of 100 - kernel weigh.

\begin{tabular}{|c|c|c|c|c|c|c|c|c|c|}
\hline \multirow{2}{*}{ Genotypes } & \multicolumn{2}{|c|}{$\mathbf{F}_{1}$} & \multicolumn{2}{|c|}{$\mathbf{F}_{2}$} & \multirow{2}{*}{ Genotypes } & \multicolumn{2}{|c|}{$F_{1}$} & \multicolumn{2}{|c|}{$\mathbf{F}_{2}$} \\
\hline & $\mathbf{N}$ & $\mathbf{L}$ & $\mathbf{N}$ & $\mathbf{L}$ & & $\mathbf{N}$ & $\mathbf{L}$ & $\mathbf{N}$ & $\mathbf{L}$ \\
\hline $\mathrm{P}_{1}$ & $0.155^{*}$ & $0.341^{* *}$ & 0.049 & $0.237 * *$ & $\mathrm{P}_{2} \mathrm{XP}_{8}$ & $0.425^{* *}$ & $0.263 * *$ & 0.233 & 0.253 \\
\hline $\mathrm{P}_{2}$ & -0.027 & $-0.243 * *$ & -0.128 & $-0.272 * *$ & $\mathrm{P}_{3} \mathrm{xP}_{4}$ & $-0.201 *$ & $0.378 * *$ & -0.455 & 0.214 \\
\hline $\mathrm{P}_{3}$ & $0.286 * *$ & 0.101 & $0.280 * *$ & $0.126^{*}$ & $\mathrm{P}_{3} \mathrm{xP}_{5}$ & $-0.404 * *$ & 0.165 & -0.184 & 0.225 \\
\hline $\mathrm{P}_{4}$ & 0.112 & $-0.161^{*}$ & $0.262 * *$ & -0.035 & $\mathrm{P}_{3} \mathrm{xP}_{6}$ & 0.004 & $0.207^{*}$ & 0.070 & -0.005 \\
\hline $\mathrm{P}_{5}$ & $0.191 * *$ & $0.298 * *$ & $0.321 * *$ & $0.357 * *$ & $\mathrm{P}_{3} \mathrm{xP}_{7}$ & $-0.284 * *$ & $-0.388 * *$ & -0.352 & 0.009 \\
\hline $\mathrm{P}_{6}$ & -0.079 & -0.068 & -0.058 & -0.001 & $\mathrm{P}_{3} \mathrm{xP}_{8}$ & $0.220^{*}$ & $0.405 * *$ & $0.832 * *$ & $0.335^{*}$ \\
\hline $\mathrm{P}_{7}$ & $-0.358 * *$ & $-0.138^{*}$ & $-0.417 * *$ & $-0.255^{* *}$ & $\mathrm{P}_{4} \mathrm{xP}_{5}$ & $-0.438 * *$ & $-0.210^{*}$ & -0.320 & -0.285 \\
\hline $\mathrm{P}_{8}$ & $-0.280^{* *}$ & $-0.130^{*}$ & $-0.311 * *$ & -0.148 & $\mathrm{P}_{4} \mathrm{XP}_{6}$ & $-0.219^{*}$ & 0.152 & 0.264 & 0.174 \\
\hline $\mathrm{P}_{1} \mathrm{XP}_{2}$ & $0.202^{*}$ & 0.086 & 0.203 & -0.124 & $\mathrm{P}_{4} \mathrm{xP}_{7}$ & 0.022 & -0.161 & 0.017 & 0.119 \\
\hline $\mathrm{P}_{1} \mathrm{xP}_{3}$ & 0.091 & 0.048 & -0.433 & -0.194 & $\mathrm{P}_{4} \mathrm{xP}_{8}$ & 0.060 & $0.450 * *$ & 0.275 & $0.380^{*}$ \\
\hline $\mathrm{P}_{1} \mathrm{XP}_{4}$ & 0.041 & $-0.262 * *$ & -0.157 & -0.054 & $\mathrm{P}_{5} \mathrm{xP}_{6}$ & $0.218^{*}$ & $-0.286 * *$ & -0.089 & -0.201 \\
\hline $\mathrm{P}_{1} \mathrm{xP}_{5}$ & 0.066 & $0.237 * *$ & -0.074 & 0.219 & $\mathrm{P}_{5} \mathrm{xP}_{7}$ & $-0.249 *$ & 0.088 & 0.117 & 0.146 \\
\hline $\mathrm{P}_{1} \mathrm{xP}_{6}$ & -0.036 & -0.036 & 0.355 & $0.537 * *$ & $\mathrm{P}_{5} \mathrm{xP}_{8}$ & $0.225^{*}$ & $0.313 * *$ & 0.347 & 0.046 \\
\hline $\mathrm{P}_{1} \mathrm{XP}_{7}$ & 0.179 & $0.911^{* *}$ & 0.400 & 0.021 & $\mathrm{P}_{6} \mathrm{xP}_{7}$ & $0.196^{*}$ & $0.341^{* *}$ & -0.179 & -0.250 \\
\hline $\mathrm{P}_{1 \mathrm{xP}} \mathrm{P}_{8}$ & $0.510 * *$ & $0.594 * *$ & 0.166 & $0.414^{*}$ & $\mathrm{P}_{6} \mathrm{xP}_{8}$ & $0.205^{*}$ & 0.145 & 0.051 & 0.044 \\
\hline $\mathrm{P}_{2} \mathrm{XP}_{3}$ & $0.229^{*}$ & -0.005 & -0.017 & -0.020 & $\mathrm{P}_{7} \mathrm{XP}_{8}$ & $0.402 * *$ & 0.113 & -0.155 & 0.171 \\
\hline $\mathrm{P}_{2} \mathrm{XP}_{4}$ & $0.286 * *$ & 0.065 & 0.359 & 0.027 & S.E. gi & 0.056 & 0.060 & 0.077 & 0.055 \\
\hline $\mathrm{P}_{2} \mathrm{XP}_{5}$ & 0.155 & $-0.221^{*}$ & 0.124 & -0.168 & S.E. sij & 0.099 & 0.090 & 0.236 & 0.168 \\
\hline $\mathrm{P}_{2} \mathrm{XP}_{6}$ & $-0.248^{*}$ & -0.027 & -0.441 & 0.083 & S.E. (gi-gj) & 0.098 & 0.183 & 0.016 & 0.083 \\
\hline $\mathrm{P}_{2} \mathrm{XP}_{7}$ & $-0.250^{*}$ & $0.420^{* *}$ & -0.239 & 0.070 & S.E. (sij-sik) & 0.295 & 0.256 & 0.349 & 0.248 \\
\hline
\end{tabular}

* and **: significant at 0.05 and 0.01 levels of probability, respectively. 


\section{Conclusion}

The study of diallel crosses of durum wheat more favorable element for the selection of these genotypes under both planting date. The result that showed highly significant negative heterosis in the tow hybrids $\left(\mathrm{P}_{3} \times \mathrm{P}_{5}\right)$ and $\left(\mathrm{P}_{4} \times \mathrm{P}_{6}\right)$ under both tow planting date. Early maturity crosses can be used in breeding program for getting early maturity lines by different selection methods. While, eight hybrid showed highly significant positive heterosis under late planting date i.e. $\left(\mathrm{P}_{1} \times \mathrm{P}_{6}\right),\left(\mathrm{P}_{2} \times \mathrm{P}_{4}\right),\left(\mathrm{P}_{2} \times \mathrm{P}_{5}\right),\left(\mathrm{P}_{2} \times \mathrm{P}_{7}\right)$, $\left(\mathrm{P}_{5} \times \mathrm{P}_{6}\right),\left(\mathrm{P}_{5} \times \mathrm{P}_{7}\right)\left(\mathrm{P}_{6} \times \mathrm{P}_{7}\right)$ and $\left(\mathrm{P}_{7} \times \mathrm{P}_{8}\right)$ for no. spike/plant, $\left(\mathrm{P}_{2} \times \mathrm{P}_{7}\right)$ and $\left(\mathrm{P}_{4} \times \mathrm{P}_{7}\right)$ for grain yield / plant, $\left(\mathrm{P}_{1} \times \mathrm{P}_{8}\right)$ and $\left(\mathrm{P}_{2} \times \mathrm{P}_{8}\right)$ for 100 -kernel weight under both tow planting date , addition these hybrid were tolerant heat stress.

$\mathrm{P}_{4}, \mathrm{P}_{7}$ showed highly significant positive GCA effects in four traits under both tow planting date. These parents could be consider as good combiner for tolerant heat stress, Also $\mathrm{P}_{2}$ showed highly positive GCA effects in three traits under both planting date, which could be unlisted in beading for ear lines in wheat.

$\mathrm{P}_{8}$ showed positive and highly significant GCA effect under two planting date. This parent could be as a good combiner for lateness. While, the cross $\left(\mathrm{P}_{3} \times \mathrm{P}_{8}\right)$ showed positive values for 100-krnel weight under both planting date

\section{References:}

Abdel-Hameed, A.S. 2002. Analysis of variance and its components of some hexaploid wheat crosses. M.Sc. Thesis Agron Dep., Fac. Agric., Minia Univ. Egypt.

Abdel-kader,M.N 2006. Genetical studies on Grain yield and some agronomic characters in some durum wheat crosses. M.Sc. Thesis, faculty of Agric. ElMinia., Univ., Egypt

Abel-Majeed, S.A., A.M Moussa and A.A. Abdel-Karim 2004. Combinig ability for yield and its components in bread wheat (Triticum aestivum L.) crosses. Egypt. J. Appl. Sci. 19(7A): 132-142.

Akinci, C. 2009. Hetetrosis and combining ability estimates in $6 \times 6$ half-diallel crosses of durum wheat (Triticum durum Desf). Bulg. J. Agric., 15: 214-221.

Al-Koddoussi, A.R. and E.E. Hassan. 1991. Heterosis in relation to general and specific combining ability in durum wheat (Triticum trurgidum, var. durum). Zagazig J. Agric. Res. 18(1): 29.

Al-Koddoussi, A.R. and E. E.Hassan. 1995. Heterosis in relation to general and specific combining ability in durum wheat (Triticum turgidium var. durum). Zagazig J. Agric. Res., 18(1) : 19.

Ashoush, H.A.; A.A. Hamada and I.H. Darwish. 2001. Heterosis and combining ability in $\mathrm{F}_{1}$ and $\mathrm{F}_{2}$ diallel crosses of wheat (Triticum aestivum L. em. Thell). Mansoura J. Agric. Res. 26(5): 2579-2592.

Bakheit, B.R., M.G. Mosaad, M.A. El-Morshidy and A.M. Tammam 1989. Inheritance of yield in some durum wheat crosses. Assuit J. of Agric. Sci.,

Bhatt, G.M. 1971. Heterotic performance and combining ability in diallel cross among spring wheat (Triticum aestivum L.) Aust J. Agric. Res .22: 359-368. 
Brahim, B. and B. Mohamed. 2014. Analysis of diallel crosses between six varieties of durum wheat in semi- arid area. African J. of Biotec., 13(2):286-293.

El-Borhamy, H.S.A. 1995. Heterosis and combining ability for some characters in wheat. M.Sc. Thesis, Fac. of Agric. Al-Azhar Univ.

El-Hennawy, M.A. 1996. Heterosis and combining ability in diallel crosses of eight bread wheat varieties. Bull. Fac. Agric. Cairo Univ., 47(3): 379-392.

El-Karamity, A.S., M.A. Salem, A.M. Tamam and M.I. Mohamed. 2007. Genetical studies on grain yield and its components in durum wheat (Triticum durum var. durum). Minia J. of Agric. Res. 5 (27): 967-985.

El-Sayed, E.M.A. 1997. Quantitative inheritance of yield and some of its contributory characters in common wheat. M.Sc. Thesis, Faculty of Agric. Menofiya Univ., Egypt.

El-Sayed, E.A.M., A.M. Tammam and S.A. Ali. 2000. Genetical studies on some bread wheat crosses (Triticum aestivum L.). Menofiya J. Agric. Res., 25(2):389-401.

El-Sayed, E.M.A. 2004. A diallel cross analysis for some quantitative characters in bread wheat (Triticum aestivum L.). Egypt J. Agric. Res., 83 (4):1665-16675.

El-Shami, M.M.; T.M. Shehab ElDin; A.H. Abd El-Latif and M.S. Sharshar. 1996. Heterosis and combining ability for grain yield and some related characters in bread wheat. J. Agric.
Sci. Mansoura Univ., 21(8): 2789-2796.

FAO 2013. (Food and Agriculture Organization of United $\mathrm{Na}$ tions). Statistical Database, online at http://www.fao.org

Griffing, B. 1956. Concept of general and specific combining ability in relation to diallel crossing systems. Aust. J. Biol . Sci., 9:463-493

Hassan, E.E. 1997. Combining ability and factor analysis in durum wheat (Triticum aestivum L.). Zagazig J. Agric. Res., 24(1): 23-36

Hamada, A.A. and M.B. Tawfelis. 2001. Genetic and graphical analysis of diallel crosses of some bread wheat (Triticum aestivum L.). J. Agric. Res. Tanta Univ. 27(4): 633-647.

Hendawy, F.A. 1994. General and specific combining ability estimates in a diallel cross of seven bread wheat varieties. Menofiya J. Agric. Res. 19(1):

Ismail, A.A.; M.A. Khalifa and K.A. Hamam. 2001. Genetic studies on some yield traits of durum wheat. II- Grain Yield and its Attributes. Assiut J. of Agric. Sci., 32(2): 121-137

Irshad, M; I.khaliq;A-S.Khan and A.Ali.2012. Genetic studies for some agronomic traits in spring wheat under heat stress. Pakistan J. of Agric. Sci., 49(1): 11-20.

Karrar, A.M.A. (1980). Performance and combining ability of durum wheat in a diallel cross. M.Sc. Thesis Fac. Agric. Minia Univ., Egypt.

Mann, S.N. Sarma. 1995 Combining ability in the F1 and F2 generations of diallel cross in macaroni 
wheat (Triticum durum Desf.). Indian J. Genet., 55(2):160-165.

Mohamed, M.M. 2007. Genetic studies on durum wheat breeding for heat tolerance. M.Sc. Thesis, faculty of Agric. El-Minia., Univ., Egypt.

Mosaad, M.G.; M.A. El-Morshidy; B.R. Bakheit and A.M. Tamam 1990. Genetical studies of some morpho-physiological traits in durum wheat crosses. Assiut $\mathrm{J}$. of Agric. Sci., 21(1): 79-94

Moustafa, M.A. 2002. Gene effect for yield and yield components for four durum wheat crosses. J. Agric. Sci. Mansoura Univ., 27(1):47-60.

Nagwa, Salem R.A 2007.Heterosis and combining ability for grain yield components of some durum wheat crosses. Egypt. Agric. Res., 85(2):563-571.

Nayem, K.A. 1994. Genetic architecture of flowering and maturity in wheat (Triticum spp.). Indian J. Genet. 54(1): 63-66.

Sharma, S.N., R.S. Sain and R.K. Sharma. 2002. Genetic control of quantitative traits in durum wheat under normal and latesowing environments. Sabrao. Jour. Of Breed. And Genet. 34(1): 35-43.

Tammam, A.M. and Y.G. Abd ElGawad. 1999. Heterosis and combining ability for bread wheat under new vally conditions. Egypt J. Appl. Sci,. 14(10):122-135.

Tawfelis, M.B. 1997. Inheritance of some physiological traits, yield and its components in durum wheat. Ph.D. Thesis, Fac. Agric. Assiut Univ., Egypt.

Tawfelis, M.B., A.A. El-Sherbieny and M.N. Abd-Elkader. 2006. Genetical studies on Grain yield and some agronomic characters in some durum wheat crosses. Egypt. J. Agric. Res., 84(5): 1479-1492.

Uma Menon and S.N. Sharma. 1997. Genetics of yield determining factors in spring wheat over environments. Indian J. Genet. 57(3): 301-306.

Wahid, A., S. Gelani, M. Ashraf and M.R. Floolad. (2007). Heat tolerance in plant :An overview. Exp. Bot., 61; 199-223

Zaied, H.M.M. 1995. Combining ability in diallel cross of wheat (Triticum aestivum L.) Ph.D. Thesis, Fac. Agric., El-Minia Univ., Egypt.

Zubair, M.; A.R. Chowdhry; I.A. Khan and A. Bakhsh. 1987. Combining ability study in bread wheat (Triticum aestivum L.). Pakistan J. Bot., 19(1): 7580. 
در اسـات ور اثية علي محصول الحبوب ومكوناته في قمح الديورم تحت الإجهاد الحراري مصطفي عمر مصطفي'، عبدالعظيم احمد إسماعيل'، عاطف أبوالوفا أحمد' و موريس بديع توفيلس' ' قسم بحوث محاصيل القمح, معهد بحوث المحاصيل الحقلية، مركز البحوث الزراعية الجيزة- مصر .

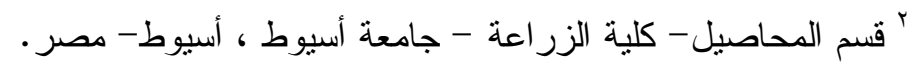

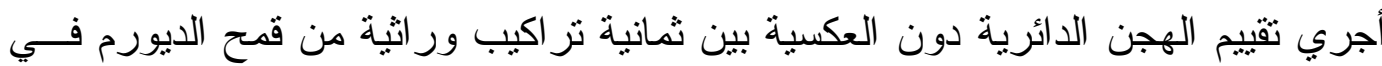

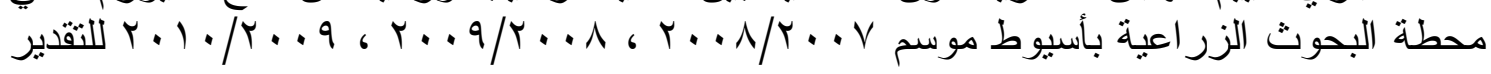

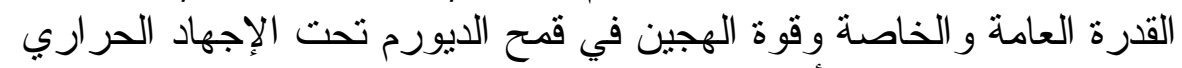

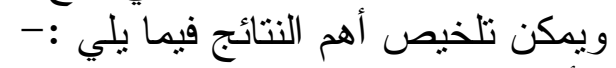

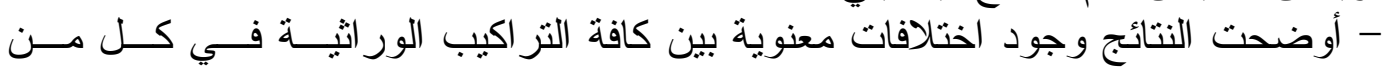

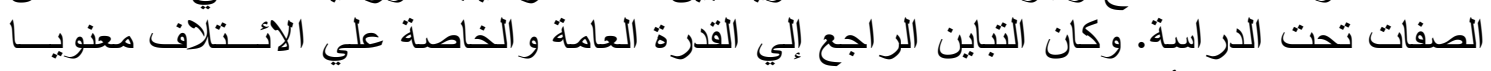

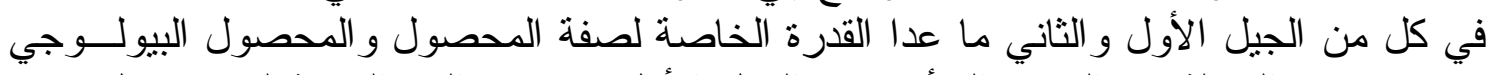

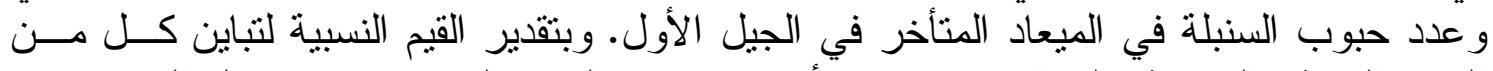

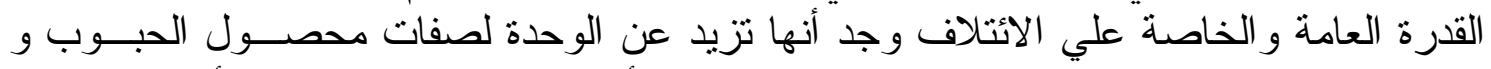

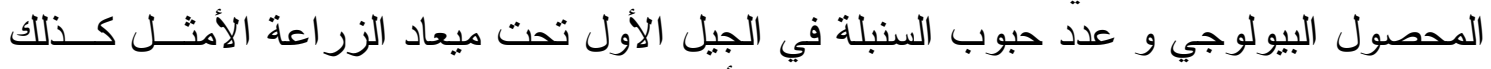

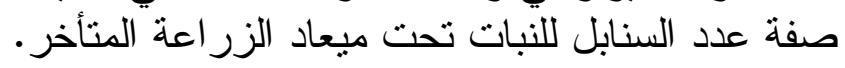

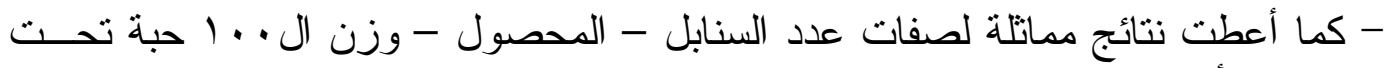

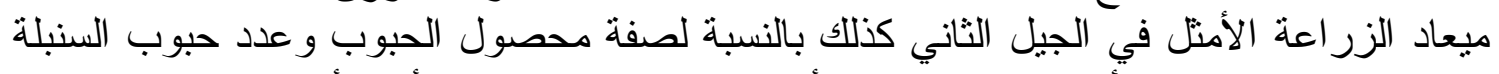

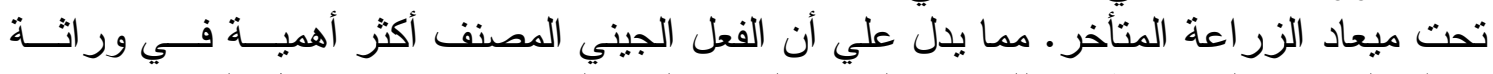

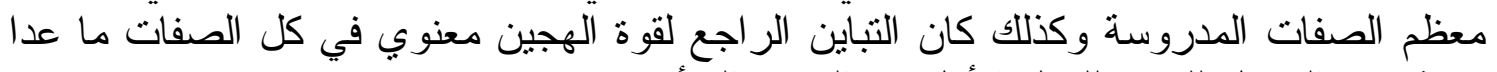

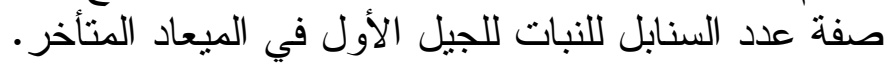

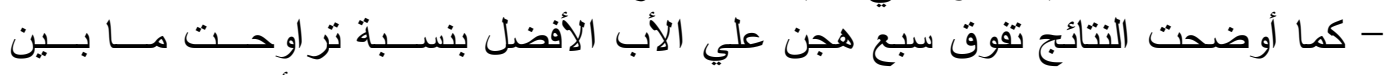

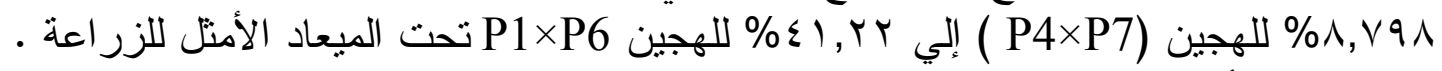

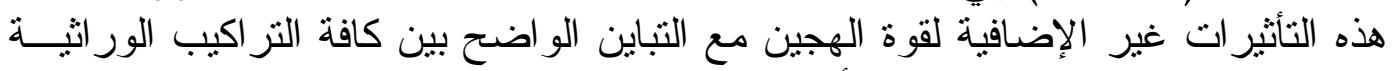

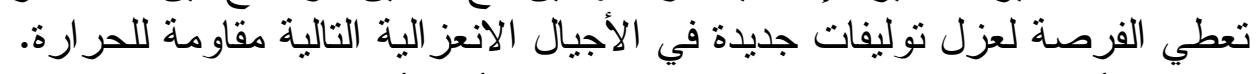

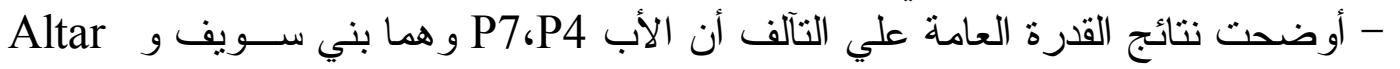

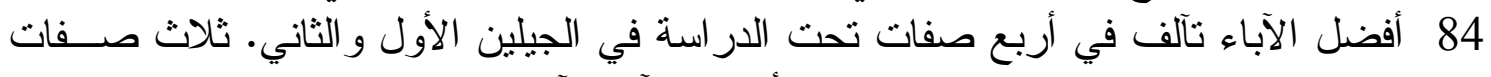

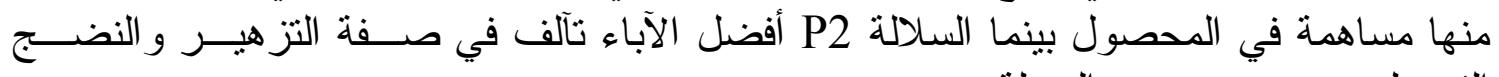

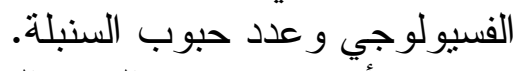

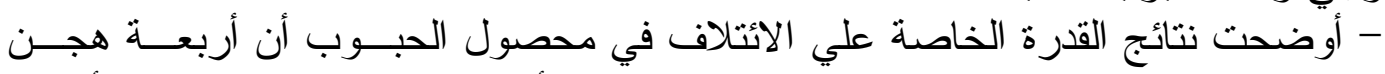

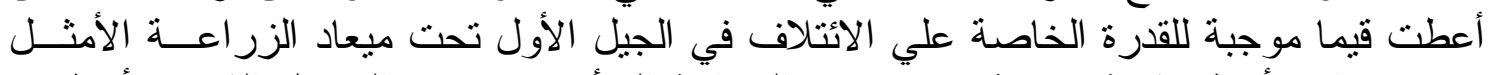

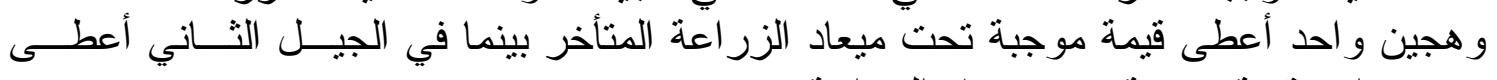

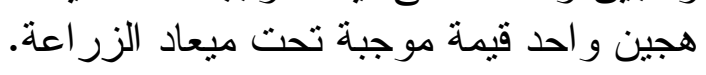

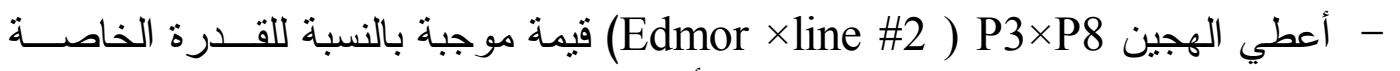

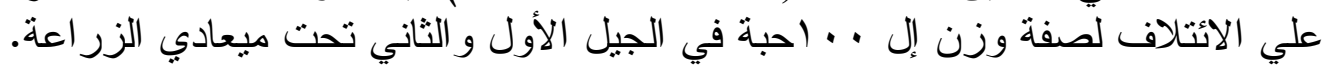

